\title{
Segmental Multi-point Linearization for Parameter Sensitivity Approximation in Reliability Analysis
}

\author{
Ke Liu ${ }^{\mathrm{a}}$, Glaucio H. Paulino ${ }^{\mathrm{a}, *}$, Paolo Gardoni ${ }^{\mathrm{b}}$ \\ ${ }^{a}$ School of Civil and Environmental Engineering, Georgia Institute of Technology, Atlanta, GA 30332, \\ United States \\ ${ }^{b}$ Dept. of Civil and Environmental Engineering, University of Illinois, Urbana, IL 61801, United States
}

\begin{abstract}
This paper proposes an efficient method named segmental multi-point linearization for accurate approximation of the sensitivity of a failure probability with respect to design parameters. The method is established based on a piecewise linear fitting of the limit state surface and the analytical integral of the gradient of the failure probability with respect to parameters in the limit state function. The proposed method presents a good ratio of accuracy to computational cost. The general framework is scalable such that, by adjusting its complexity, different levels of accuracy can be achieved. The method is especially suitable to be implemented in gradient-based routines for solving reliability-based design optimization problems where accuracy of the parameter sensitivity is essential for convergence to an optimal solution.
\end{abstract}

Keywords: reliability analysis, sensitivity analysis, reliability-based design optimization, parameter sensitivity of failure probability

\section{Introduction}

The primary concern of reliability analysis is the approximation of the failure probability $P_{f}$ or the equivalent generalized reliability index $\beta$ which, is defined as $\beta=\Phi^{-1}\left(1-P_{f}\right)$ [1], where $\Phi^{-1}(\cdot)$ is the inverse function of cumulative distribution function (CDF) of the standard normal distribution. In addition, computation of sensitivity of failure probability to changes in parameters that influence the failure probability is also an important aspect as

\footnotetext{
${ }^{*}$ Corresponding author

Email addresses: kliu89@gatech.edu (Ke Liu), paulino@gatech.edu (Glaucio H. Paulino), gardoni@illinois.edu (Paolo Gardoni)
} 
this quantification provides insights on how the failure probability might change with changes in the values of some parameters. The partial derivatives of failure probability with respect to these parameters are called parameter sensitivity [1]. The parameters are categorized into two groups [2]. One group contains the parameters that appear in the probability density functions (PDF) of random variables and they are usually called distribution parameters. The other set of parameters, called design parameters, appear in the limit state function, a mathematical description that defines whether the component or system is safe or not. In this paper, we propose an efficient numerical method to approximate the parameter sensitivity of the failure probability with respect to design parameters.

The motivation of this work is due to the increasing demand for improvement of engineering designs based on reliability analysis, for example, either reducing cost without sacrificing reliability or simply increasing reliability of current design, which can be expressed mathematically as an optimization problem [11], called reliability-based design optimization (RBDO). In the RBDO formulation, the aforementioned design parameters are treated as design variables. Most RBDO problems are formulated as optimization problems with probabilistic constraint(s). The sensitivity of the probabilistic constraint is particularly important if one wants to use efficient gradient-based routines to solve the design optimization problem.

There are mainly two major gradient-based approaches for RBDO in the literature: the Reliability Index Approach (RIA), which explicitly uses the gradient of probabilistic constraint(s) in the optimization; and the Performance Measure Approach (PMA), which constructs target performance function(s) as equivalent deterministic constraint(s) by inverse reliability analysis [12], and thus the gradient of probabilistic constraint(s) is involved implicitly. Among different alternatives, currently the two approaches are mostly implemented in conjunction with first-order reliability method (FORM) and FORM-based expressions for sensitivity [13, 14, 15, 16, 17, 18, 19. To improve the solutions, heuristic updates of the failure probability using, for example, second-order reliability method (SORM), Monte Carlo simulation (MCS), and other reliability methods, are used in many RBDO approaches [19, 16, however, in general, the approximations of the sensitivity of failure probability in these approaches are still based on FORM. Another type of gradient-based approach employs the sample average approximation (SAA) where the failure probability and its sensitivity 
calculation are both carried out using MCS-based techniques [6], which require very high computational cost but could yield quite accurate estimation of the sensitivity.

Although numerical estimation of failure probability has been studied extensively, there are not many papers in the literature that focus on the numerical approximation of the parameter sensitivity. Early work can be found around the year 1990, mostly from a theoretical point of view. For instance, Hohenbinchler and Rackwitz [2] derived the expressions for parameter sensitivity of the estimated failure probability obtained by FORM with respect to both distribution and design parameters. In other words, the expressions are only exact when the limit state function is linear. Breitung [3] derived an analytical expression for the parameter sensitivity of failure probability with general limit state functions, and he suggested an asymptotic approximation, which has similar terms as the expression in [2]. There is also a study on the derivatives of probability functions done by Uryasev [4]. However, although the analytical expressions for the parameter sensitivity of the failure probability is available, usually precise numerical evaluation of the sensitivity is not possible, similarly to the case of failure probability evaluation.

Some numerical methods have been developed to approximate the parameter sensitivity. An efficient method is available by implementing the expressions in [2], which are by nature an approximation. The advantage is that it is so simple that it can be computed with very low computational cost. However, the expressions can be quite inaccurate when the nonlinearity of the limit state function is significant. Karamchandani and Cornell [5] developed a method that approximates the parameter sensitivity with respect to distribution parameters that can take second order effect into account, based on SORM and the finite difference method. In $\underline{\text { addition, MCS-based stochastic methods are often used for approximating the sensitivity of }}$ probability [6, 7, 8, 9, 10]. For example, the sample average approximation was used for both component and system reliability constrained problems by Royset and Polak [6, 8, 9].

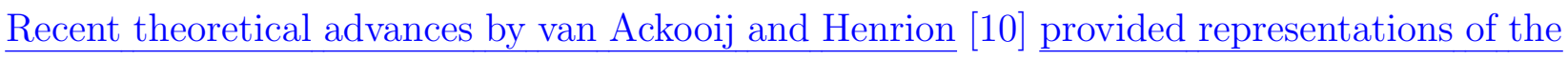
$\underline{\text { gradients to convex probability functions as integrals with respect to uniform distribution }}$ over the unit sphere.

In this paper, we focus on the approximation of the parameter sensitivity of a component failure probability since this is the foundation for extensions to system reliability analysis. 
The proposed method of segmental multi-point linearization (SML) is established to estimate the sensitivity of failure probability with respect to design parameters. The method can be directly employed in the framework of RIA for RBDO. Thus the main purpose of this work is to provide an alternative method, that is more accurate than FORM-based approximation, but requires significantly less computational cost than approximations based on MCS, as illustrated by Fig. 1. Furthermore, the theory for the method allows many variations to match the different requirements on accuracy for different problems. This paper is tailored towards the implementation of the proposed method in the context of RBDO. However, we would like to mention that the method for sensitivity analysis is independent from this particular application. The nomenclature used in this paper is given in Appendix A.

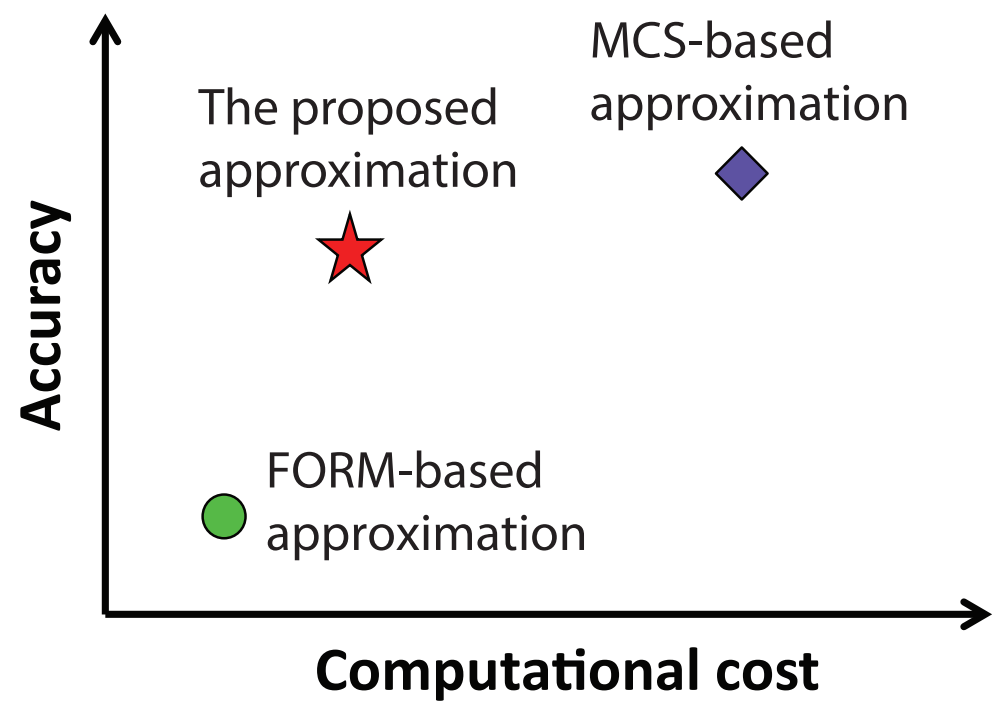

Figure 1: Conceptual comparison of different approximation approaches for sensitivity of failure probability.

\section{Importance of an accurate estimation of sensitivity in RBDO}

One problem with FORM-based approaches in RBDO is that the error in the approximate sensitivity of the probabilistic constraint can lead the optimization to converge to non-optimal solutions. This will be investigated by analyzing two popular approaches of RBDO, namely FORM-based RIA and PMA, via the Karush-Kuhn-Tucker (KKT) optimality conditions of the approaches. Consider a generic formulation of RBDO problems with one reliability 
component:

$$
\begin{gathered}
\min _{\mathbf{x}} f(\mathbf{x}) \\
\text { s.t. } \quad P_{f}=\int_{g(\mathbf{v}, \mathbf{x})<0} f_{\mathbf{v}}(\mathbf{v}) \mathrm{d} \mathbf{v} \leqslant P_{f}^{t} \\
\mathbf{h}(\mathbf{x}) \leqslant \mathbf{0}
\end{gathered}
$$

where $P_{f}^{t}$ is the target failure probability; $\mathbf{x}$ is the vector of design variables (i.e. design parameters); $\mathbf{v}$ is the vector of random variables with distribution described by the PDF $f_{\mathbf{v}}(\mathbf{v}) ; f(\mathbf{x})$ is the objective function; $g(\mathbf{v}, \mathbf{x})$ is the limit state function; and $\mathbf{h}(\mathbf{x})$ is a set of deterministic constraints such as lower and upper bounds of $\mathbf{x}$. Reliability methods often require a probability preserving transformation $\mathbf{u}=T(\mathbf{v})$, where $\mathbf{u}$ is a vector of independent standard normal random variables. Replacing $\mathbf{v}$ by $T^{-1}(\mathbf{u})$ defines the limit state function in an equivalent form $G(\mathbf{u}, \mathbf{x})=g\left(T^{-1}(\mathbf{u}), \mathbf{x}\right)$, which is the function that is eventually used for reliability analysis. The formulation (1) would accordingly become:

$$
\begin{gathered}
\min _{\mathbf{x}} f(\mathbf{x}) \\
\text { s.t. } \quad P_{f}=\int_{G(\mathbf{u}, \mathbf{x})<0} \varphi_{n}(\mathbf{u}) \mathrm{d} \mathbf{u} \leqslant P_{f}^{t} \\
\mathbf{h}(\mathbf{x}) \leqslant \mathbf{0}
\end{gathered}
$$

where $\varphi_{n}(\cdot)$ is the multi-variate standard normal PDF with uncorrelated elements and $n$ is the number of random variables. Equivalently, the constraint on failure probability can be expressed in terms of generalized reliability index, which is defined as $\beta=\Phi^{-1}\left(1-P_{f}\right)$.

Mathematically, the KKT optimality conditions of the RBDO model described in (2), are:

(1) Stationarity condition: $\nabla_{\mathbf{x}} f+\lambda \nabla_{\mathbf{x}} P_{f}+\sum \gamma_{i} \nabla_{\mathbf{x}} h_{i}=0$

(2) Primal feasibility: $P_{f}-P_{f}^{t} \leqslant 0, h_{i} \leqslant 0 \forall i$

(3) Dual feasibility: $\lambda \geqslant 0, \gamma_{i} \geqslant 0 \forall i$

(4) Complementary slackness: $\lambda\left(P_{f}-P_{f}^{t}\right)=0, \gamma_{i} h_{i}=0 \forall i$ 
where $\lambda$ and $\gamma_{i}$ 's are the Lagrange multipliers. The KKT conditions are necessary for a solution to be optimal. Because for most RBDO cases, $P_{f}$ and $\nabla_{\mathbf{x}} P_{f}$ can only be evaluated approximately, then the KKT conditions are only approximately satisfied at the optimized solution. Different RBDO algorithms have different approximations about the KKT conditions in the end. Thus they may converge to different solutions. However, when the probabilistic constraint is active in an optimization problem, PMA and RIA will converge to the same solution if they are both based on FORM as they share the same approximations of the KKT conditions.

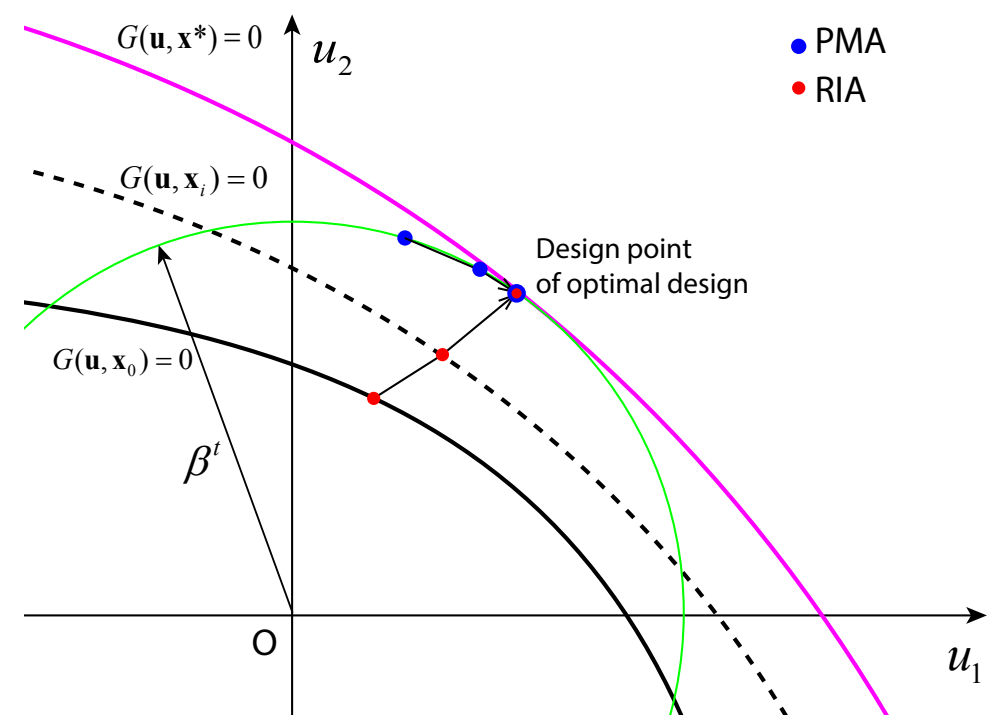

Figure 2: Trajectory of the optimization process of RIA and PMA in the standard normal random space.

In the RIA formulation, the reliability constraint is considered directly. The sensitivity 110 of failure probability with respect to design variables is used to obtain the search direction in optimization. The expression for the gradient $\nabla_{\mathbf{x}} \beta_{1}$ is derived by Hohenbichler and Rackwitz [2]:

$$
\nabla_{\mathbf{x}} \beta_{1}=\frac{1}{\left\|\nabla_{\mathbf{u}} G^{*}\right\|} \nabla_{\mathbf{x}} G^{*}
$$

where $\beta_{1}=\left\|\mathbf{u}^{*}\right\|$ which is the approximate reliability index by FORM; and $G^{*}$ denotes $G\left(\mathbf{x}, \mathbf{u}^{*}\right)$, which is the limit state function evaluated at the design point $\mathbf{u}^{*}$ as defined by Eq. 
(4) with current design $\mathrm{x}$, where it is evaluated.

$$
\mathbf{u}^{*}=\underset{\mathbf{u}}{\operatorname{argmin}}\{\|\mathbf{u}\| \mid G(\mathbf{u}, \mathbf{x})=0\}
$$

As $P_{f}=1-\Phi(\beta)$, then we have $\mathrm{d} P_{f} / \mathrm{d} \beta=-\varphi(\beta)$. By applying the chain rule, we obtain:

$$
\nabla_{\mathbf{x}} P_{f} \approx \nabla_{\mathbf{x}} P_{f, 1}=-\frac{\varphi\left(\beta_{1}\right)}{\left\|\nabla_{\mathbf{u}} G^{*}\right\|} \nabla_{\mathbf{x}} G^{*}
$$

where the notation $P_{f, 1}$ denotes the FORM approximation. The KKT stationarity condition of FORM-based RIA is then given by:

$$
\nabla_{\mathbf{x}} f+\lambda^{R I A}\left[-\frac{\varphi(\beta)}{\left\|\nabla_{\mathbf{u}} G^{*}\right\|} \nabla_{\mathbf{x}} G^{*}\right]+\sum \gamma_{i} \nabla_{\mathbf{x}} h_{i}=0
$$

As stated before, Eq. (5) is the sensitivity of the approximate failure probability obtained by FORM [13]. In general, differentiation of an approximate expression enlarges the error [25]. Therefore, even in the cases that FORM provides a good approximation of $P_{f}$, the sensitivity $\nabla_{\mathbf{x}} P_{f}$ calculated by FORM-based approximation could have a relatively large error.

The FORM-based PMA formulation applies an inverse FORM reliability analysis. The approach defines a target performance function $G^{t}(\mathbf{x})=G\left(\mathbf{x}, \mathbf{u}^{t}\right)$ and incorporates it as a deterministic constraint of the optimization, where $\mathbf{u}^{t}$ is an estimation of the design point of the optimal design, which is also called the most probable point (MPP), and is updated at each iteration as:

$$
\mathbf{u}^{t}=\underset{\mathbf{u}}{\operatorname{argmin}}\left\{G(\mathbf{u}, \mathbf{x}) \mid\|\mathbf{u}\|=\beta^{t}=\Phi^{-1}\left(1-P_{f}^{t}\right)\right\}
$$

Usually an approximation of the MPP $\mathbf{u}^{t}$ is used to reduce computational cost. The advantage of PMA is that it is not sensitive to the accuracy of $\mathbf{u}^{t}$. As a result, many single loop algorithms, which involve a coarse approximation of $\mathbf{u}^{t}$, are developed based on PMA [14, 16]. However, when the optimum is achieved and the probabilistic constraint become active, $\mathbf{u}^{t}$ converges to the design point of the final design [12]. The KKT stationarity 
condition of the PMA becomes:

$$
\nabla_{\mathbf{x}} f+\lambda^{P M A}\left(-\nabla_{\mathbf{x}} G^{*}\right)+\sum \gamma_{i} \nabla_{\mathbf{x}} h_{i}=0
$$

Equations (6) and (8) are the same, except for the different coefficients of the second term. If the probabilistic constraint is active and the design point is unique, the two approaches should yield:

$$
\lambda^{P M A}=\frac{\varphi(\beta)}{\left\|\nabla_{\mathbf{u}} G^{*}\right\|} \lambda^{R I A}
$$

Given that the probability of failure are both approximated by FORM, the KKT conditions of RIA and PMA become identical. Hence, although PMA tends to be more robust than RIA, it does not improve the result of the optimization because it is mathematically equivalent to RIA when the problem has active probabilistic constraint and the corresponding design point is unique. If we look into the iteration process, the $\mathbf{u}^{*}$ for RIA and $\mathbf{u}^{t}$ for PMA are at different coordinates until they converge to the same design. The trajectories of RIA and PMA in the random space are illustrated in Fig. 2, where $\mathbf{x}^{*}$ refers to the "optimal" design, $\mathbf{x}_{0}$ is the initial design, and $\mathbf{x}_{i}$ represent an intermediate design in the optimization process.

Many algorithms, which are developed based on RIA and PMA, incorporate SORM, MCS or other reliability methods to improve the approximation of $P_{f}$ (i.e. the primal feasibility condition) [19, 23, but little attention has been paid to the accuracy of the sensitivity which may be more influential with respect to the optimal solution. Furthermore, the error in the sensitivity is cumulative because it determines the search direction at each iteration of gradient-based optimization schemes.

\section{Segmental multi-point linearization for approximating sensitivity and failure probability}

References [3] and [4] provide the analytical expression for the sensitivity of failure probability with respect to design parameters, which has the following form:

$$
\nabla_{\mathbf{x}} P_{f}=-\int_{S} \frac{\varphi_{n}(\mathbf{u})}{\left\|\nabla_{\mathbf{u}} G\right\|} \nabla_{\mathbf{x}} G \mathrm{~d} S
$$

For the sake of completeness and clarity, we provide a brief derivation of Eq. 10] in Appendix

B. In most cases, the integral in Eq. 10 is not numerically tractable because it is a multi- 
dimensional surface integral. We propose an efficient numerical method for the approximation of this surface integral. As motivated by the aforementioned discussion, the method requires much less computational effort than MCS techniques and the accuracy of the approximation is higher than FORM-based approximations (i.e. Eq. (3) and (5) - see Fig. 1.

Calculation of the surface integral of Eq. (10) is challenging both analytically and numerically. However, if the surface is composed of a set of pieces of hyperplanes described by affine functions, then Eq. (10) can be analytically simplified to probability evaluation problems. Thus the idea of the proposed method is to fit the limit state surface with hyperplane segments in a piecewise manner. Then we can perform the integration on each plane segment without much effort and compute the summation of the individual integrals as the approximation of the sensitivity in Eq. (10).

Let's denote the limit state surface as $S$ and the piecewise linear fitting as $\bar{S}$ where each of the hyperplane segments is denoted as $\bar{S}_{i}$. For each hyperplane segment $\bar{S}_{i}$, which is assumed to be described by an affine function $\bar{G}_{i}$, the gradients $\nabla_{\mathbf{u}} \bar{G}_{i}$ and $\nabla_{\mathbf{x}} \bar{G}_{i}$ will be constant vectors over the corresponding surface. Thus, they can be taken out of the integral, which leads to the following expression:

$$
\nabla_{\mathbf{x}} P_{f, i}=-\frac{1}{\left\|\nabla_{\mathbf{u}} \bar{G}_{i}\right\|} \nabla_{\mathbf{x}} \bar{G}_{i} \int_{\bar{S}_{i}} \varphi_{n}(\mathbf{u}) \mathrm{d} \bar{S}_{i}
$$

Since all fitting segments are pieces of hyperplanes, each hyperplane segment naturally has piecewise linear boundaries, thus its geometry appears as polygons. To further simplify Eq. (11), for each of the hyperplane segments, we can then rotate the coordinates of the standard normal space such that the positive direction of the first axis is along the opposite direction of the normal direction of the hyperplane segment. Because the function that defines the hyperplane is linear, the normal direction of the hyperplane is in the same direction of $\nabla_{\mathbf{u}} \bar{G}$, as shown in Fig. 3. Due to the rotational symmetry of the standard normal space, we can rewrite Eq. (11) by separating the new coordinate $u_{1}^{\prime}$ from the integral:

$$
\nabla_{\mathbf{x}} P_{f, i}=-\frac{\varphi\left(b_{i}\right)}{\left\|\nabla_{\mathbf{u}} \bar{G}_{i}\right\|} \nabla_{\mathbf{x}} \bar{G}_{i} \int_{\bar{S}_{i}} \varphi_{n-1}\left(\hat{\mathbf{u}}^{\prime}\right) \mathrm{d} \hat{\mathbf{u}}^{\prime}
$$

where $b_{i}$ is the distance from the origin to the hyperplane, and $\hat{\mathbf{u}}=\left[u_{2}^{\prime}, u_{3}^{\prime}, \ldots, u_{n}^{\prime}\right]^{\mathrm{T}}$. The surface integral on one of the hyperplane segments is then simplified to the probability evalu- 


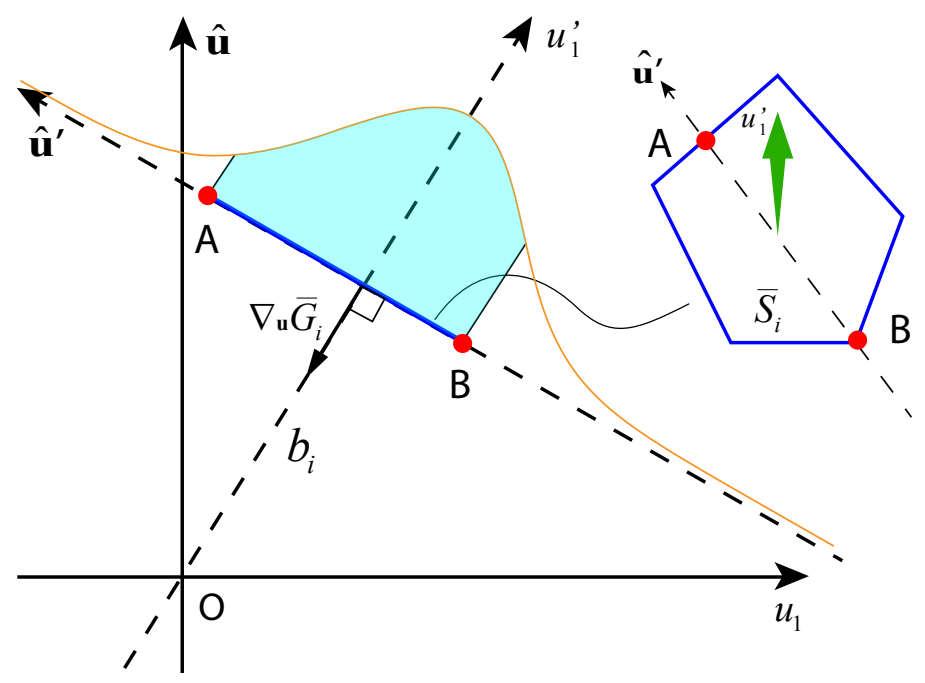

Figure 3: Illustration of the calculation of Eq. 10 on a hyperplane segment.

ation of $\int_{\bar{S}_{i}} \varphi_{n-1}(\hat{\mathbf{u}}) \mathrm{d} \hat{\mathbf{u}}^{\prime}$ where the integration domain is composed of a polygonal hyperplane segment that spans in the reduced $(n-1)$ dimensional space of $\hat{\mathbf{u}}^{\prime}$. Assuming that we have a proper piecewise linear fitting of the limit state surface where each piece of hyperplane segments is representative of a portion of the limit state surface, then we are able to construct an approximation of $\nabla_{\mathbf{x}} P_{f}$, based on Eq. (12), that has the form of the following weighted sum:

$$
\nabla_{\mathbf{x}} P_{f} \approx \sum_{i=1}^{p} W_{i} \nabla_{\mathbf{x}} \bar{G}_{i}
$$

where

$$
W_{i}=-\frac{\varphi\left(b_{i}\right)}{\left\|\nabla_{\mathbf{u}} \bar{G}_{i}\right\|} \int_{\bar{S}_{i}} \varphi_{n-1}\left(\hat{\mathbf{u}}^{\prime}\right) \mathrm{d} \hat{\mathbf{u}}^{\prime}
$$

Notice that the rotated new coordinate system $\mathbf{u}^{\prime}$ is different for each hyperplane segment.

A proper fitting scheme is essential to the approximation. In general, each hyperplane segment can be completely defined by a fitting point, the normal direction of the hyperplane, and the boundary of the segment. A fitting point is a point that is on the original limit state surface where the fitting hyperplane segment has to pass. It is important to notice that, due to the exponential decay of the probability density in the standard normal space, we only need to focus on the region that is close to the origin where the probability density is high. According to Eq. 10, the integrand becomes too small to make an impact on the overall integration when it is evaluated far from the origin. Also, as we increase the number of fitting 
hyperplane segments, the accuracy of this approximation can be improved.

\subsection{Tangent Fitting Scheme}

In order to approximate the limit state surface, a straightforward thought is to let $\bar{G}_{i}$ be the first order expansion of the limit state function $G$ at the corresponding fitting point $\mathbf{u}_{i}$, which ensures that, in the neighborhood, the linear approximation of the original limit state surface is quite accurate. That is,

$$
\nabla_{\mathbf{x}} \bar{G}_{i}=\nabla_{\mathbf{x}} G\left(\mathbf{u}_{i}, \mathbf{x}\right) \quad \text { and } \quad \nabla_{\mathbf{u}} \bar{G}_{i}=\nabla_{\mathbf{u}} G\left(\mathbf{u}_{i}, \mathbf{x}\right)
$$

where $\mathbf{x}$ is the vector of current design variables. The boundaries of a segment are determined by the intersections with other segments. This leads to a tangent fitting scheme as shown in Fig. 4. In particular, it should be noted that if we use only one hyperplane to fit the limit state surface by taking the tangent fitting at the design point, then from Eq. (13) and (14) we can obtain Eq. (5), indicating that the FORM approximation can be seen as a special case of the proposed method. If the locally most central points are selected to be the fitting points, the tangent fitting share the same approximation of the limit state surface as the multi-point FORM [1], but here the approximation of the limit state surface is primarily used to estimate the sensitivity of the failure probability rather than the failure probability itself.

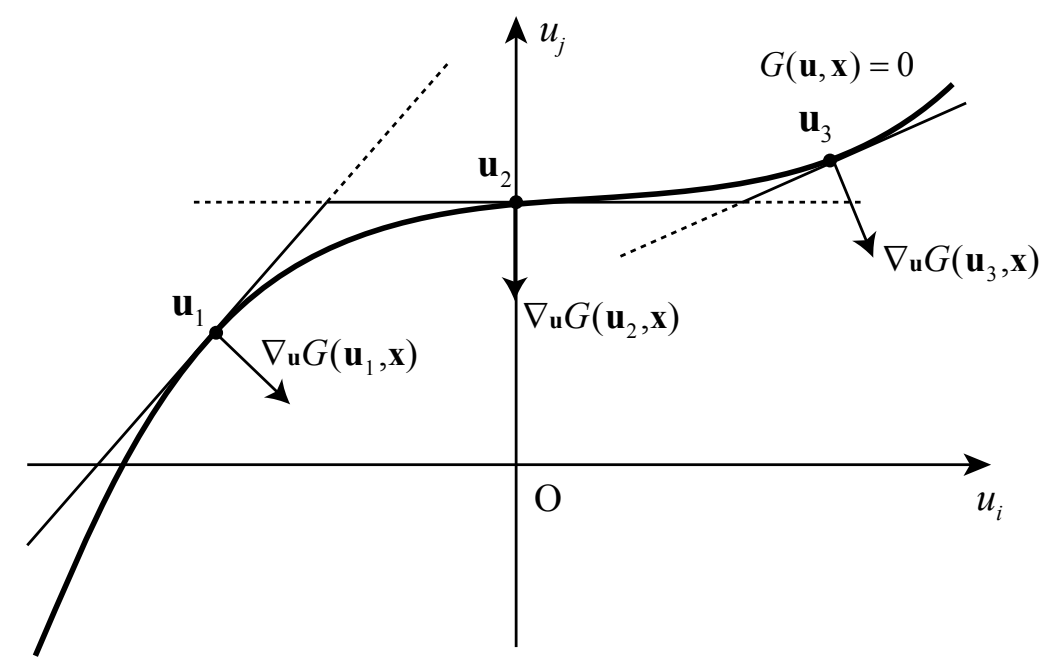

Figure 4: Illustration of alternative tangent fitting SML.

However, in high dimensional random space, the tangent fitting scheme makes it quite difficult to track the boundaries of the hyperplane segments. To overcome this challenge, 
we can prescribe the normal directions of the hyperplane segments and then project the gradient $\nabla_{\mathbf{u}} G$ of the original limit state function onto the prescribed normal directions as the gradients $\nabla_{\mathbf{u}} \bar{G}_{i}$ 's for the linear approximations. Since the design variables $\mathbf{x}$ are not in the random space of $\mathbf{u}$, the term of $\nabla_{\mathbf{x}} \bar{G}_{i}$ in Eq. (14) will not be affected by this projection in the random space and thus we can still obtain first order accuracy for $\nabla_{\mathbf{x}} G$. In other words, to define the affine function $\bar{G}_{i}$ for each hyperplane segment, we take

$$
\nabla_{\mathbf{u}} \bar{G}_{i}=\left(\mathbf{n}_{i}^{\mathrm{T}} \nabla_{\mathbf{u}} G\left(\mathbf{u}_{i}, \mathbf{x}\right)\right) \mathbf{n}_{i}
$$

where $\mathbf{n}_{i}$ is the prescribed normal direction for the $i$ th plane segment, and keep $\nabla_{\mathbf{x}} \bar{G}_{i}$ as in the tangent fitting scheme. Hence, this compromise only affect the computation of the weights, however, for a hyperplane segment with fitting point $\mathbf{u}_{i}$, the angle between the gradient $\nabla_{\mathbf{u}} G\left(\mathbf{u}_{i}, \mathbf{x}\right)$ and normal direction $\mathbf{n}_{i}$ should not be too large in a relative sense, otherwise the weights can be misleading. For example, in an extreme case, if the two vectors are orthogonal to each other, the corresponding weight will be infinitely large. There are many alternative ways to specify the normals and, different choices of the normals lead to different fitting schemes.

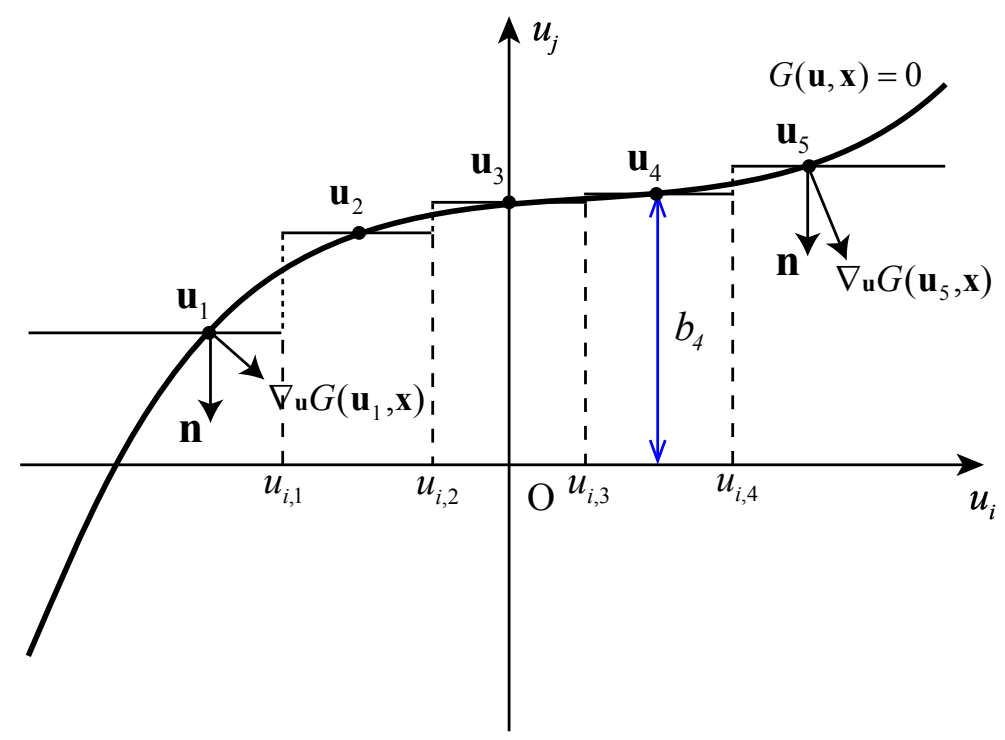

Figure 5: Illustration of alternative step fitting SML. 


\subsection{Step Fitting Scheme}

Another attempt for a proper fitting scheme is the step fitting. We can fit the limit state surface by a piecewise "constant" function where each hyperplane segment basically has the same normal direction. Also due to the exponential decay of probability density, only the limit state function that is in the central region would be fitted with the step function. Fig. 5 illustrates the idea of the step fitting scheme. One can choose how many fitting points to use and prescribe the boundaries by defining the length of each side of the step segments. The normals of the step segments need not to be in the direction of any of the original axes of the $\mathbf{u}$-space. Rotation of the standard normal random space is allowed, for example, to make the rotated first axis align with the direction of the design point. In addition, this scheme would simplify the probability evaluation part in the calculation of the weights to closed form expressions because the edges of each plane segment are perpendicular to each other. For example, in Fig. 5, if there are only two random variables such that $u_{i}=u_{1}$ and $u_{j}=u_{2}$, the weight for plane segment 4 is simply given by

$$
W_{4}=\varphi\left(b_{4}\right)\left(\Phi\left(u_{1,4}\right)-\Phi\left(u_{1,3}\right)\right) /\left\|\nabla_{\mathbf{u}} G\left(\mathbf{u}_{4}, \mathbf{x}\right) \mathbf{n}\right\|
$$

Furthermore, this fitting scheme is scalable. If we increase the number of fitting points, i.e., make each step plane segment sufficiently small, and evaluate the probability integration part in Eq. (11) using MCS, we can recover the MCS-based estimation expression of $\nabla_{\mathbf{x}} P_{f}$ as proposed in reference [6]. The problem with this scheme is that sometimes the result can be quite inaccurate due to a potentially large angle between $\left.\nabla_{\mathbf{u}} G\left(\mathbf{u}_{i}, \mathbf{x}\right)\right)$ and $\mathbf{n}$.

\subsection{Orthogonal Fitting Scheme}

Another scheme that is recommended is called orthogonal fitting. The basic idea is to fit the limit state surface with plane segments that have normals along an orthogonal basis of the space instead of sharing the same normal direction as in the step fitting to avoid large angles between $\left.\nabla_{\mathbf{u}} G\left(\mathbf{u}_{i}, \mathbf{x}\right)\right)$ and $\mathbf{n}_{i}$. The general procedure is described in Table. 1 and illustrated in Fig. 6:

The values for $k_{1}$ and $k_{2}$ are determined based on heuristic rules. The parameter $k_{1}$ determines the size of the search region for the intersection point, and it is suggested to have 
Table 1: Procedure for the orthogonal fitting scheme

1. Select a reference point on the limit state surface (possibly but not necessarily the design point)

2. Rotate the coordinates such that the reference point lies on the positive part of the first axis of the new coordinates

3. Search for the intersection points of the new axes and the limit state surface within radius $r=k_{1} b_{1}$ from the origin in both positive and negative directions, where $k_{1}$ is a user defined parameter and $b_{1}$ is the distance from the reference point to the origin

4. Define the plane segment $i$ by its fitting point with the normal $\mathbf{n}_{i}$ being the direction of the axis on which the fitting point lies

5. For the half axis $\pm \mathbf{e}_{j}^{\prime}$, that has no intersection point within the search region, fit a plane segment with normal direction along $\mathbf{e}_{1}^{\prime}$ direction at the off-axis point $\mathbf{u}_{j}$ (denote as $\mathbf{u}_{j+n}$ for $-\mathbf{e}_{j}^{\prime}$ ) with coordinate $\pm k_{2} b_{1} \mathbf{e}_{j}^{\prime}+b_{j} \mathbf{e}_{1}^{\prime}$

the value such that $\varphi\left(k_{1} b_{1}\right) / \varphi\left(b_{1}\right)=\epsilon$ where $\epsilon$ is a small value (e.g., $\left.\epsilon=0.1\right)$ to ensure that that the off-axis fitting points stay not too far from the origin but also not too close to the reference point. In general, we recommend to set $k_{2}$ as the minimum between 1 and $3 / b_{1}$, which is the same rule as in the point-fitting SORM proposed by Der Kiureghian et al. [24], except that the reference point is not necessarily the design point. In addition, a partition coefficient $\eta$ is used to determine the boundaries of the plane segments determined by the reference point and the off-axis fitting points. In practice, the value of $\eta$ should take a value between 0.5 and 1.0 in order to take into account the exponential decay of probability density from the origin. In this paper, 0.7 is used for all numerical examples. The rotation in step (2) can be accomplished using any QR factorization method, like the Gram-Schmidt algorithm [25]. After all the parameters and the new orthogonal basis are defined, finding the fitting points is only a problem of solving 1-D nonlinear equations. There are many efficient and robust algorithms available, such as bisection method [25]. A pseudo-code for the segmental multi-point linearization with orthogonal fitting scheme is given in Appendix C. 


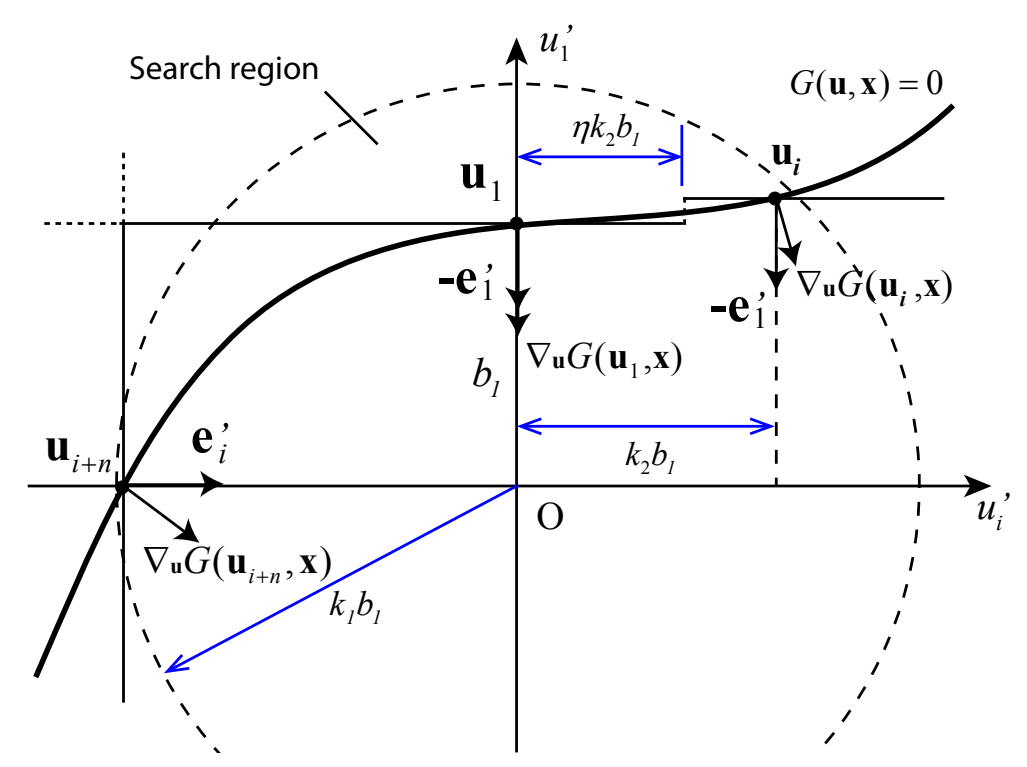

Figure 6: Illustration of alternative orthogonal fitting SML.

\subsection{Orthogonal Fitting Remarks}

The choice of the reference point influences accuracy of the SML approximation obtained by the orthogonal fitting scheme. Intuitively, the reference point should be close to the origin. The design point is a good candidate for the reference point. However, in some particular cases, other choices of the reference point would actually make the approximation more accurate. For example, when the limit state surface has a symmetry axis that passes through the origin, then it would be beneficial to set the reference point along this symmetry axis. This is due to the fact that, when the limit state surface is symmetric, according to Eq. (10), the two halves of the limit state surface on both sides of the symmetry axis are of same importance to the integral. A reference point on the symmetry axis makes the fitting points symmetrically distributed on the two halves and hence, in the approximation, the two halves of the limit state surface are also of same importance. Another question is whether performing a second rotation of the random space in step (2), such that the fitting points are along the principle axes of curvature, would be beneficial to accuracy. The second rotation is the same as the second rotation in the traditional SORM. This will be elaborated in the second numerical example. One shortcoming of this fitting scheme is that it is not scalable with the current formulation. The number of fitting points is always $2 n$ or $(2 n-1)$, where $n$ is the dimension of the random space. Thus, adding more fitting points to increase the 
accuracy of the approximation is not automatically supported by this scheme. Sometimes the off-axis fitting points can be neglected, for instance, when the limit state function appears like a sphere or ellipsoid. This corresponds to a reduced version of the orthogonal fitting scheme 26 .

\subsection{Approximation of Failure Probability}

Each fitting scheme provides an approximation of $P_{f}$, which is consistent with the approximated limit state surface. Based on existing approximation of the limit state surface, computing the approximate failure probability is a relatively light task. The output is observed to be generally better than FORM. Thus, one can approximate both the gradient of the failure probability and the failure probability itself using the SML method. Alternatively, one may also couple other reliability methods, for example SORM, point-fitting SORM and MCS with the SML method for approximating the failure probability, and only use SML for approximating the sensitivity of failure probability.

\section{Numerical examples}

Several numerical examples are provided to assess the various features of the SML method. The examples proposed are the following:

1. Investigation of different methods for sensitivity approximation;

2. Investigation of orthogonal fitting SML and assessment of the second rotation of the random space;

3. Design optimization of a reinforced concrete girder;

4. Application to reliability-based topology optimization.

In the first example, we compare various methods to estimate the gradient of failure probability (e.g. FORM, MCS, and SML) and demonstrate different fitting schemes of the proposed SML method. The second example addresses the orthogonal fitting SML and assesses the second rotation of the random space (see Table. 2). The third example is a practical structural problem dealing with the optimal design of a reinforced concrete girder, which was adopted from Royset and Polak [6]. Finally, the fourth example presents an application of 
the SML method to reliability-based topology optimization on discrete structures discretized

\subsection{Investigation of different methods for sensitivity approximation}

In this example, we make a comparison of a variety of methods to estimate the gradient of failure probability, namely, FORM (i.e. Eq. (5)), MCS employed in SAA [6], and the proposed SML method. We also demonstrate various fitting schemes of the SML method in this example.

Consider a limit state function that is defined in the standard normal space:

$$
G\left(x, u_{1}, u_{2}\right)=3-u_{2}-x u_{1}^{2}
$$

where $x$ is the design parameter; $u_{1}$ and $u_{2}$ are random variables. The constant 3 is selected to make the failure probability $P_{f}=P(G<0)$ around 0.0013 , which is a typical value for failure probability in engineering design problems. This limit state function is an exemplary quadratic limit state function, which has also been used as benchmark problems in other papers [24, 29].

The fitting schemes being compared are tangent fitting (TF) scheme, step fitting (SF) scheme and orthogonal fitting (OF) scheme, as described in previous section. The fitting points for the tangent fitting scheme are taken as the same as the fitting points for the orthogonal fitting scheme with the reference point along the symmetry axis. For the SML method with step fitting, the fitting points are chosen to be 9 points that are equally spaced in $u_{1}$ from -4.5 to 4.5. Each step segment has the same length except the two end step segments which are extended to infinity. The orthogonal fitting scheme is implemented with two variations: one takes the reference point at a fixed point $(0,3)$ which is along the symmetry axis of the limit state curve; the other takes the design point as the reference point. The improved HLRF algorithm [30] is employed to find the design point. Notice that when $x$ becomes large, there are two symmetric design points for this example, however, as a numerical optimization algorithm, the improved HLRF algorithm is only able to find one of them.

Fig. 7 shows the approximations of $\mathrm{d} P_{f} / \mathrm{d} x$ by the different methods for different values 
of $x$. The exact solution is obtained by direct numerical integration of Eq. (10), which can be done in this example since limit state surface is a one dimensional curve. In most application, it is very difficult to perform the numerical integration. The Monte Carlo estimation is conducted with 5000 simulations. The corresponding estimation of generalized reliability index $\beta$ is also plotted in Fig. 8. Similarly, the exact solution is obtained by direct numerical integration.

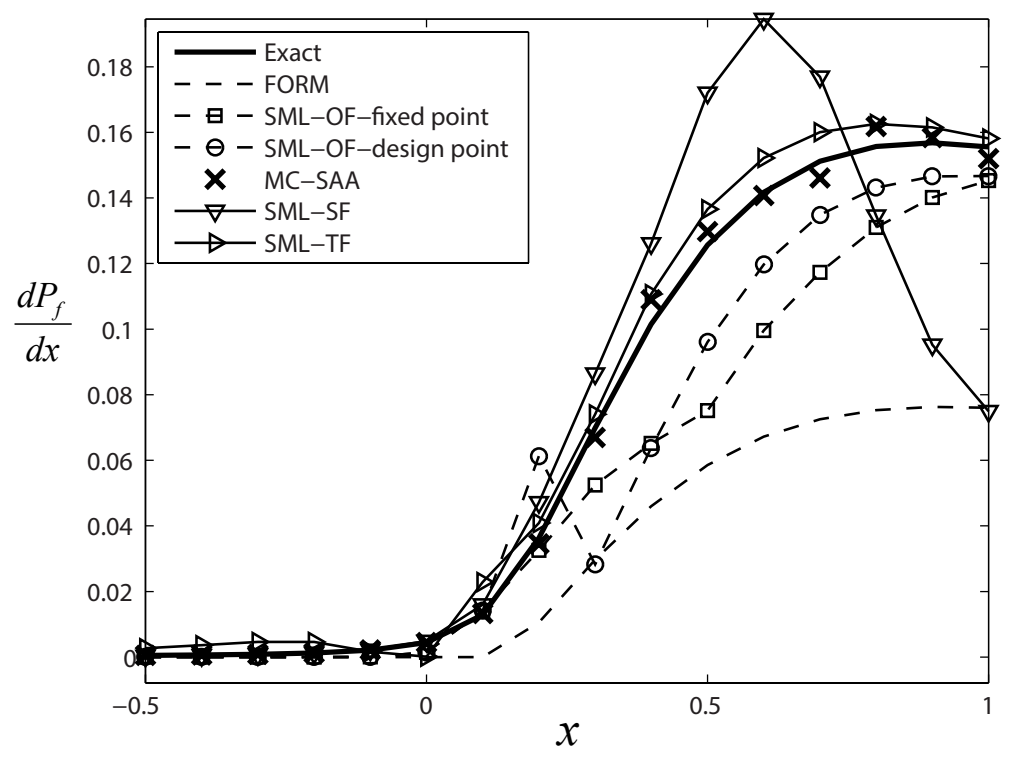

Figure 7: Variation of the gradient of failure probability with respect to $x$.

One can observe that in this example, FORM cannot provide accurate approximations for the sensitivity $\mathrm{d} P_{f} / \mathrm{d} x$. The MCS employed in the sample average approximation is quite accurate for both $\mathrm{d} P_{f} / \mathrm{d} x$ and $\beta$, but it is expensive in terms of computational cost. The approximated $\mathrm{d} P_{f} / \mathrm{d} x$ by step fitting SML matches the exact value quite well when $x<0.4$. The error then becomes large due to the increasing angle between the $\nabla_{\mathbf{u}} G$ at the fitting points and the prescribed normal of plane segments. As expected, the tangent fitting SML provides the best approximation about $\mathrm{d} P_{f} / \mathrm{d} x$ among all fitting schemes of the SML method, with an accuracy comparable to the estimation by MCS. The two orthogonal fitting schemes are both relatively accurate in general. Although not as accurate as the tangent fitting, the approximation are much simpler to construct especially in high dimensional random space. The jump in the curve for the orthogonal fitting with fixed reference point is due to the 


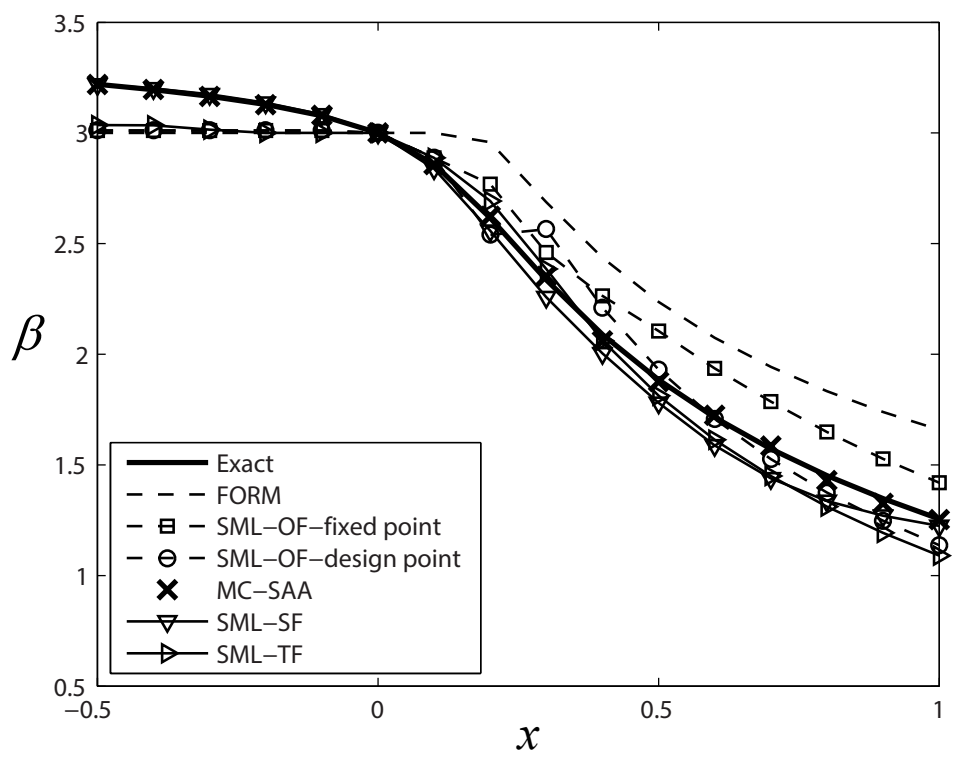

Figure 8: Variation of the reliability index with respect to $x$.

sudden switch of the fitting points from off-axis points to intersection points as the curvature becomes large. Both of the two orthogonal fitting schemes provide better approximation about $P_{f}$ than FORM. Since the fixed reference point $(0,3)$ is on the symmetry axis of the limit state surface, the orthogonal fitting scheme with fixed reference point has a better overall accuracy than the other variation.

\subsection{Investigation of orthogonal fitting SML}

This example is to examine the effect of the aforementioned second rotation on the accuracy of SML estimation. We consider four limit state functions defined with original random variables denoted as $v_{1}, v_{2}$ and $v_{3}$ :

$$
\begin{aligned}
& g_{1}(\mathbf{v}, \mathbf{x})=x_{3}-v_{3}-x_{2} v_{2}^{2}-x_{1} v_{1}^{2} \\
& g_{2}(\mathbf{v}, \mathbf{x})=x_{3}-v_{3}-x_{2} v_{2}^{2}-x_{1} v_{1}^{2}+0.1 \sin \left(10 v_{2}\right) \sin \left(10 v_{3}\right) \\
& g_{3}(\mathbf{v}, \mathbf{x})=x_{3}-v_{3}-x_{2} v_{2}^{2}-0.2 x_{1} v_{1}^{3} \\
& g_{4}(\mathbf{v}, \mathbf{x})=x_{3}-v_{3}-x_{2} v_{2}^{2}-0.1 x_{1} v_{1}^{4}
\end{aligned}
$$

where $x_{1}, x_{2}$ and $x_{3}$ are the design variables. In this example, the random variables have the same marginal standard normal distribution, and $v_{2}$ and $v_{3}$ are correlated with a correlation 


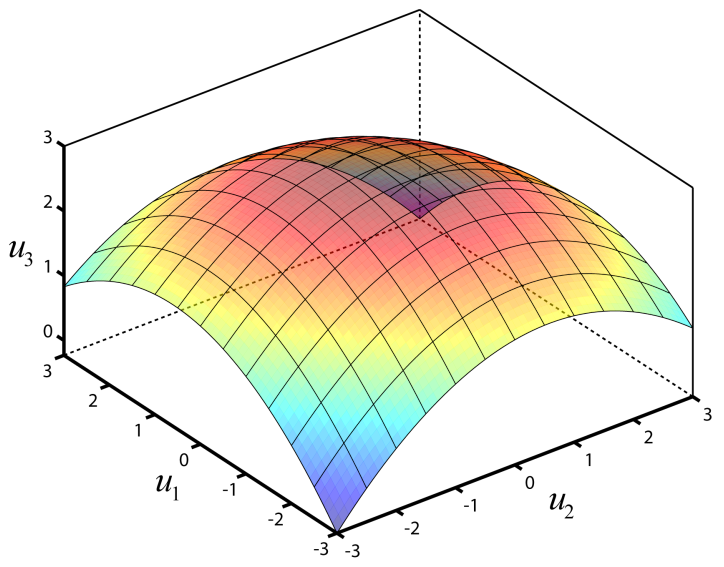

(a)

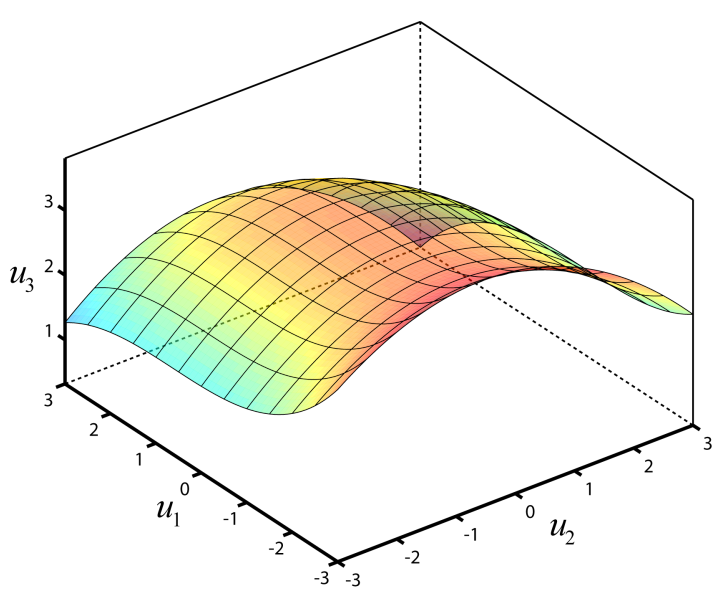

(c)

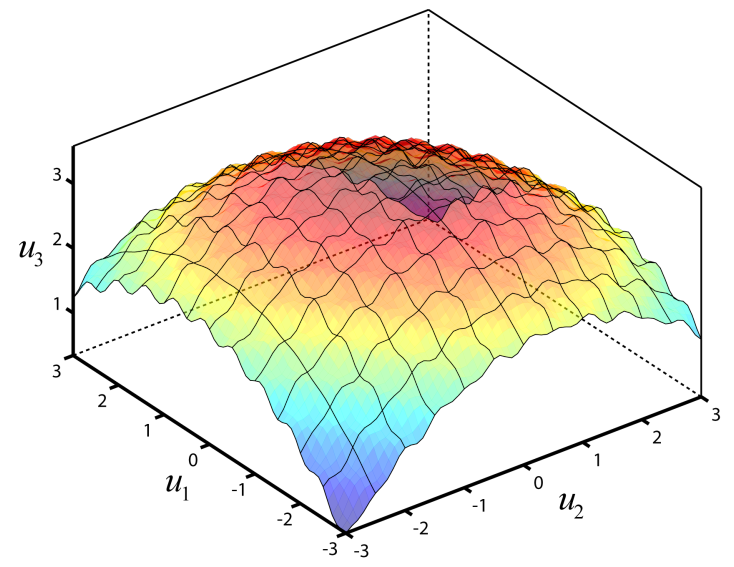

(b)

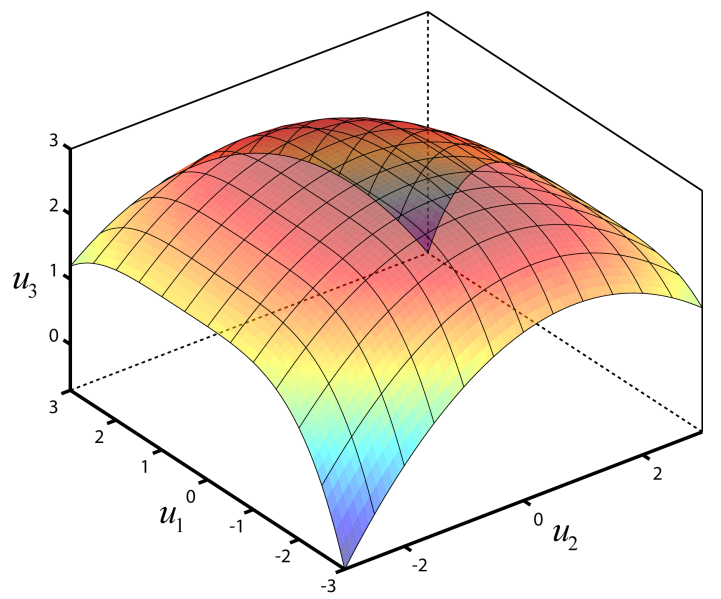

(d)

Figure 9: (a) $G_{1}(\mathbf{u}, \mathbf{x})=0$. (b) $G_{2}(\mathbf{u}, \mathbf{x})=0$. (c) $G_{3}(\mathbf{u}, \mathbf{x})=0$. (d) $G_{4}(\mathbf{u}, \mathbf{x})=0$.

coefficient 0.2. In function $g_{2}$, we introduce an artificial sinusoidal "noise" following similar manner as in the paper in which the point-fitting SORM is proposed [24]. The coefficients of 0.2 and 0.1 that applies to the last term of $g_{3}$ and $g_{4}$ are just to moderate the steep change of value for high order polynomial functions such that the four limit state functions yield similar failure probability but have different shapes of limit state surfaces. The value of $x_{2}$ is taken as 0.15 and $x_{3}=3.0$. Various values of $x_{1}$ are tested in the investigation. Fig. 9 depicts the corresponding limit state surfaces in the standard normal random space after transformation of the random variables for the case $x_{1}=0.15$.

The difference in the two orthogonal fitting SML methods is that for one of them we just do the standard procedure as described in Section 3, however, for the other one, after step 
(2), we perform another rotation that makes the new coordinates to be along the principle axes of curvature at the reference point. This can be done by doing eigenvalue analysis of the Hessian of the limit state function evaluated at the reference point. Since we select the design point as the reference point in this example, the second rotation will be the same as the one in the traditional SORM. The obtained results by the orthogonal fitting scheme with 1 rotation and 2 rotations are also compared with the approximated gradient obtained by FORM. The comparisons are made through the relative angle $\alpha$ between the approximation and the gradient estimated by MCS as in [6] using 25000 Monte Carlo simulations which is expected to be very close to the actual gradient. We use the maximum number of simulations as suggested in the paper [6] which, equals 25000. In optimization, the direction of an approximate gradient is the main concern about its accuracy, since most modern optimization algorithms employ techniques to adaptively determine the step size, which means that the norm of the gradient is not as important as its direction in an optimization. Therefore, the angle $\alpha$ serves as a good measure of accuracy in the approximation of gradient of failure probability with respect to design variables for optimization applications. The smaller the angle, the more accurate the approximation is. Fig. 10a shows the values of $\alpha$ for limit state function $g_{1}$ with different values of $x_{1}$. For limit state function $g_{2}, g_{3}$, and $g_{4}$, the comparisons of $\alpha$ are shown in Fig. 10b, 10c, 10d. From the result, we can see that the two orthogonal fitting SML methods have overall similar errors. The second rotation does not show a significant benefit to the accuracy of the approximation. In addition, since the second rotation depends on local information of the limit state function, we can observe that accuracy of the orthogonal fitting SML with 2 rotations was affected by the "noise" in the limit state function as shown in Fig. 10b. Thus, given that the second rotation is obtained by computationally expensive eigenvalue analysis of the Hessian matrix, the implementation of the second rotation in SML method is not included in the proposed method. The overall accuracy of the approximation obtained by standard orthogonal fitting SML method is significantly better than the FORM-based approximation. For most optimization problems, the accuracy of the sensitivity approximation by the orthogonal fitting SML should be sufficient to lead to convergence of the optimization to the neighborhood of a real optimum.

The approximations of $P_{f}$ are also given as shown in Fig. 11 in terms of reliability index 


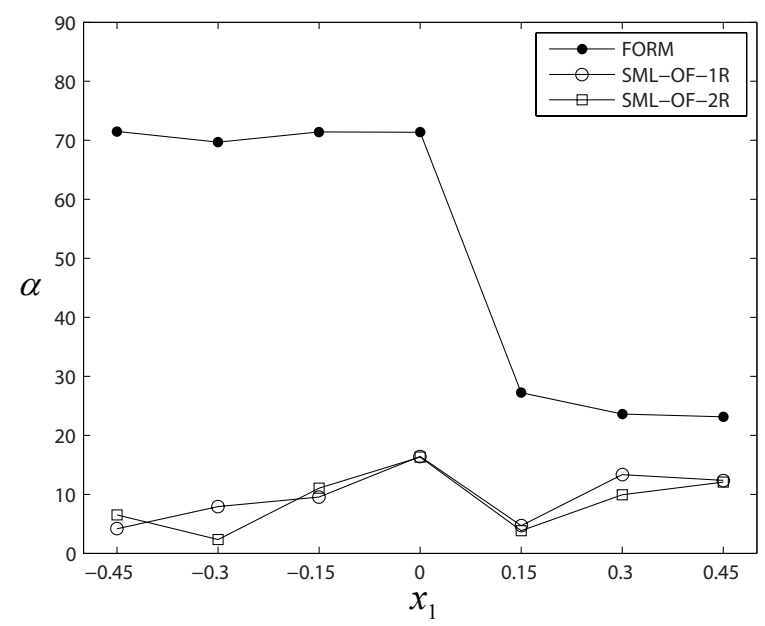

(a)

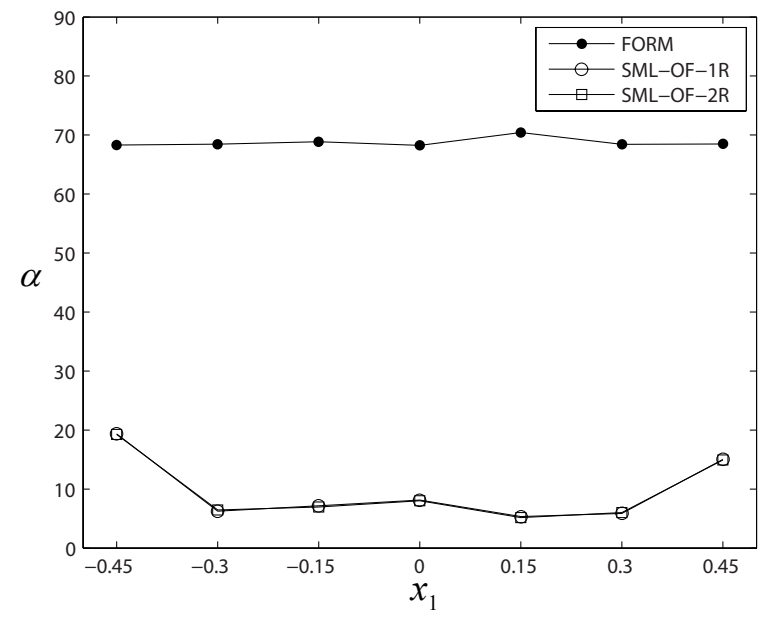

(c)

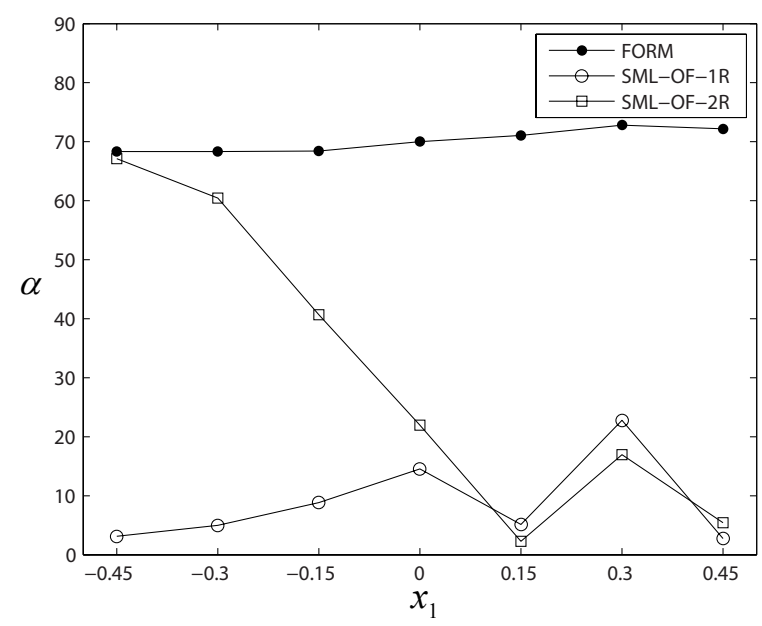

(b)

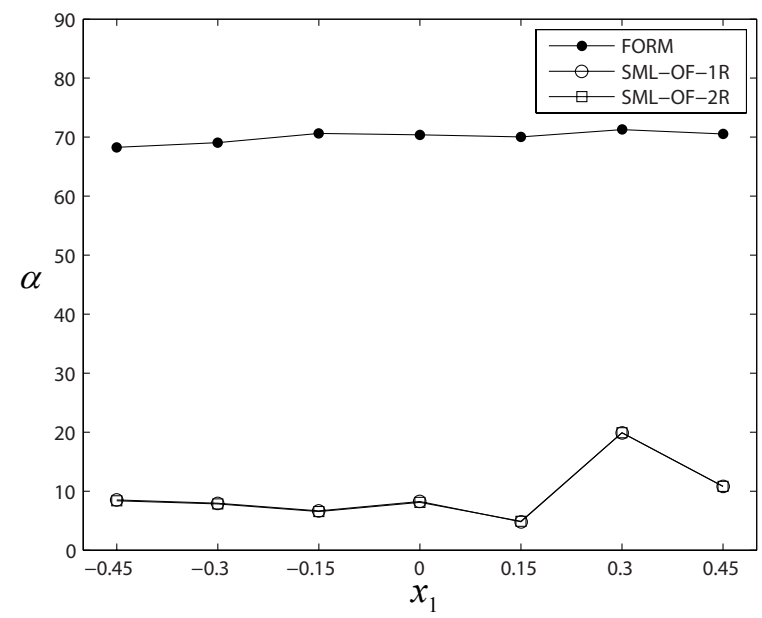

(d)

Figure 10: The measured angle $\alpha$ between the approximate gradient and the actual gradient of $P_{f}$ with respect to $\mathbf{x}$ for: (a) $g_{1}\left(G_{1}\right)$, (b) $g_{2}\left(G_{2}\right)$, (c) $g_{3}\left(G_{3}\right)$, (d) $g_{4}\left(G_{4}\right)$.

B. FERUM [31] is used to perform FORM, SORM, point-fitting SORM (PFSORM), and plain Monte Carlo simulation in this example. The plain MCS is conducted with c.o.v. of 0.05 (not a fixed number of simluations) since we want a rational estimation of the failure probability as a standard reference for the comparison, and the numbers labeled in the figures shows the number of simulations performed for the plain MCS to achieve the required c.o.v.. The numerical values can be found in Table. 2 for the case $x_{1}=0.15$. For SORM and point fitting SORM, only the approximations calculated by Tvedt integration is plotted in Fig. 11, as the Breitung's asymptotic formula might fail to be evaluated under some 
negative curvatures [32]. The result indicates that the point-fitting SORM would be a good choice for approximating the failure probability that can be easily coupled with the SML method if it is used to estimate the sensitivity only. The point-fitting SORM and SML could also share some of the fitting points, such that we can save computational cost. Besides, the approximation of $P_{f}$ by the SML method is also generally accurate and better than traditional FORM. Depending on the requirements on the accuracy of probability estimation, one can choose any applicable methods for approximation of the failure probability. For the sake of robustness and efficiency, the author would suggest that in a design optimization problem, one should use the SML for both approximation of $\nabla_{\mathbf{x}} P_{f}$ and $P_{f}$ until the optimization is close to convergence, and then use other reliability methods such as point-fitting SORM and MCS to refine the approximation of $P_{f}$. A good indication of the time to switch can be the time when the change in the objective of the optimization becomes small among iterations.

Table 2: Comparison of different reliability analysis methods for limit state functions at $x_{1}=0.15$

\begin{tabular}{|c|c|c|c|c|c|c|c|c|c|c|}
\hline \multirow{3}{*}{ Case } & \multirow{3}{*}{ Parameter } & \multicolumn{3}{|c|}{ No Rotation } & \multicolumn{3}{|c|}{1 Rotation } & \multicolumn{3}{|c|}{2 Rotations } \\
\hline & & Plain MCS & MCS-SAA & & PFSC & RM & & SOR & & \\
\hline & & $($ c.o.v. $=0.05)$ & $(\mathrm{N}=25000)$ & FORM & Breitung & Tvedt & SML-OF & Breitung & Tvedt & SML-OF \\
\hline \multirow{3}{*}{$g_{1}$} & $\beta$ & 2.50 & 2.52 & 2.99 & - & 2.51 & 2.59 & 2.48 & 2.61 & 2.75 \\
\hline & $\alpha$ & NA & $0^{\circ}$ & $27.24^{\circ}$ & NA & NA & $4.68^{\circ}$ & NA & NA & $3.84^{\circ}\left(48.69^{\circ} *\right)$ \\
\hline & $\left\|\nabla_{\mathbf{x}} P_{f}\right\|$ & NA & $5.09 \mathrm{e}-02$ & $6.08 \mathrm{e}-03$ & NA & NA & $4.88 \mathrm{e}-02$ & NA & NA & $3.13 \mathrm{e}-02$ \\
\hline \multirow{3}{*}{$g_{2}$} & $\beta$ & 2.52 & 2.53 & 2.90 & 2.37 & 2.51 & 2.59 & 3.83 & 3.87 & 2.52 \\
\hline & $\alpha$ & NA & $0^{\circ}$ & $71.08^{\circ}$ & NA & NA & $5.12^{\circ}$ & NA & NA & $2.29^{\circ}\left(140.87^{\circ}\right)$ \\
\hline & $\left\|\nabla_{\mathbf{x}} P_{f}\right\|$ & NA & $4.95 \mathrm{e}-02$ & $5.88 \mathrm{e}-03$ & NA & NA & $4.36 \mathrm{e}-02$ & NA & NA & $5.31 \mathrm{e}-02$ \\
\hline \multirow{3}{*}{$g_{3}$} & $\beta$ & 2.74 & 2.74 & 3.00 & 2.62 & 2.74 & 2.74 & 2.63 & 2.75 & 2.74 \\
\hline & $\alpha$ & NA & $0^{\circ}$ & $69.71^{\circ}$ & NA & NA & $6.52^{\circ}$ & NA & NA & $6.38^{\circ}\left(89.99^{\circ}\right)$ \\
\hline & $\left\|\nabla_{\mathbf{x}} P_{f}\right\|$ & NA & $2.46 \mathrm{e}-02$ & $4.43 \mathrm{e}-03$ & NA & NA & $3.36 \mathrm{e}-02$ & NA & NA & $3.30 \mathrm{e}-02$ \\
\hline \multirow{3}{*}{$g_{4}$} & $\beta$ & 2.64 & 2.65 & 3.00 & 2.39 & 2.57 & 2.65 & 2.63 & 2.75 & 2.65 \\
\hline & $\alpha$ & NA & $0^{\circ}$ & $70.04^{\circ}$ & NA & NA & $4.80^{\circ}$ & NA & $\mathrm{NA}$ & $4.91^{\circ}\left(90.00^{\circ}\right)$ \\
\hline & $\left\|\nabla_{\mathbf{x}} P_{f}\right\|$ & NA & $3.04 \mathrm{e}-02$ & $4.43 \mathrm{e}-03$ & NA & NA & $3.85 \mathrm{e}-02$ & NA & NA & $3.82 \mathrm{e}-02$ \\
\hline
\end{tabular}

*Note: This angle measures the rotation angle of second rotation after the first rotation of the random space.

\subsection{Design of reinforced concrete girder}

This example adopted from Royset and Polak [6] deals with the optimal design of a reinforced concrete girder. Consider a simply supported highway bridge that is made by reinforced 


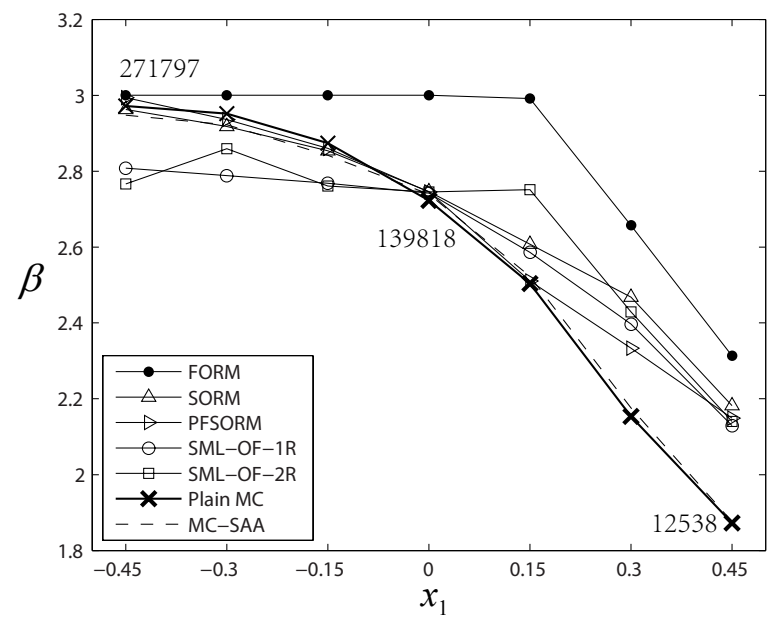

(a)

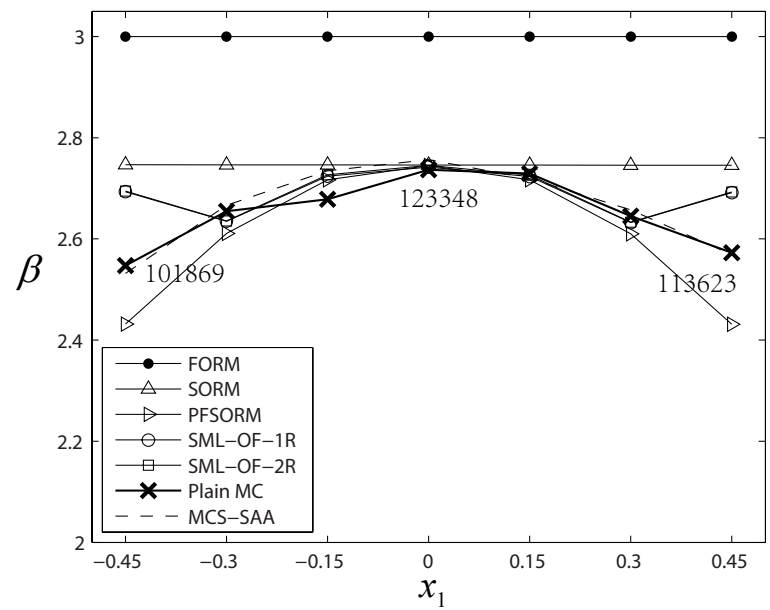

(c)

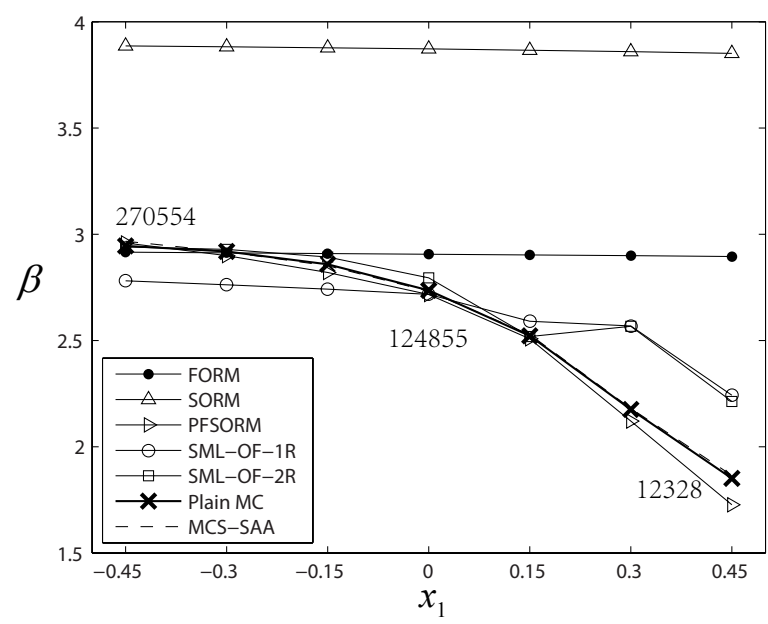

(b)

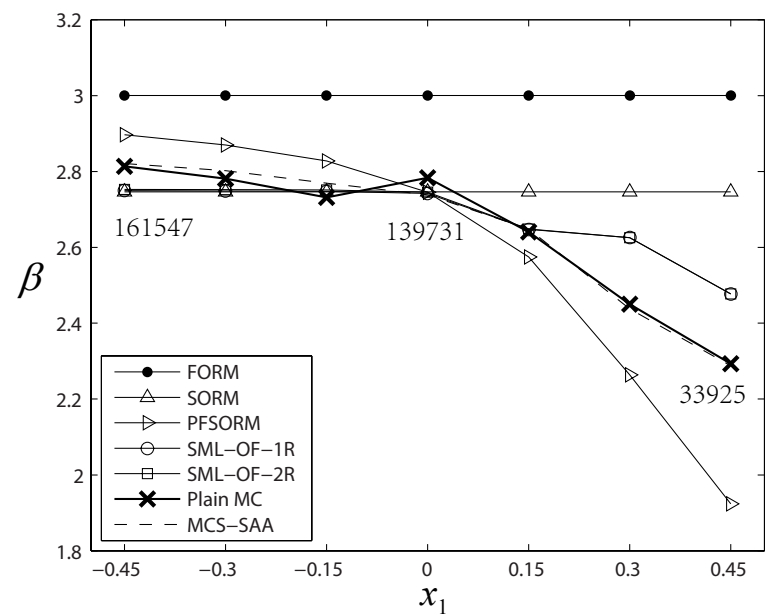

(d)

Figure 11: Comparison of the obtained generalized reliability index $\beta=\Phi^{-1}\left(P_{f}\right)$ using different methods, and the numbers are the number of simulations performed by FERUM for $x_{1}=-0.45, x_{1}=0.0$, and $x_{1}=0.45$. (a) $g_{1}\left(G_{1}\right)$, (b) $g_{2}\left(G_{2}\right),\left(\right.$ c) $g_{3}\left(G_{3}\right),\left(\right.$ d) $g_{4}\left(G_{4}\right)$.

concrete with a T-shaped cross section as shown in Figs. 12 and 13 . The material and load data are the same with the original example in [6]. Detailed description of the problem can be found in [6] and [33]. The design variables are $\mathbf{x}=\left[A_{s}, b, h_{f}, b_{w}, h_{w}, A_{v}, S_{1}, S_{2}, S_{3}\right]$, where $A_{s}$ is the area of longitudinal reinforcement, $b$ and $h_{f}$ are the width and thickness of the flange respectively, $b_{w}$ and $h_{w}$ are the width and depth of the web, $A_{v}$ is the area of the vertical reinforcement which equals twice the cross section area of each stirrup, and $S_{1}, S_{2}$ and $S_{3}$ are the three different spacings of the stirrups. The original random variables are 
$\mathbf{v}=\left[f_{y}, f_{c}^{\prime}, P_{D}, M_{L}, P_{S 1}, P_{S 2}, P_{S 3}, W\right]$ as defined in Table. 3 .

Table 3: Statistics of normal random variables

\begin{tabular}{cccc}
\hline Variables & Description & Mean & $\begin{array}{c}\text { Coefficient } \\
\text { of variation }\end{array}$ \\
\hline$f_{y}(\mathrm{~Pa})$ & $\begin{array}{c}\text { Yield strength } \\
\text { of reinforcement }\end{array}$ & $413.4 \times 10^{6}$ & 0.15 \\
$f_{c}^{\prime}(\mathrm{Pa})$ & $\begin{array}{c}\text { Compressive strength } \\
\text { of concrete }\end{array}$ & $27.56 \times 10^{6}$ & 0.15 \\
$P_{D}(\mathrm{~N} / \mathrm{m})$ & Dead load excluding weight & $13.57 \times 10^{3}$ & 0.20 \\
$M_{L}(\mathrm{~N} \cdot \mathrm{m})$ & Live load bending moment & $929 \times 10^{3}$ & 0.243 \\
$P_{S 1}(\mathrm{~N})$ & Live load shear in interval 1 & $138.31 \times 10^{3}$ & 0.243 \\
$P_{S 2}(\mathrm{~N})$ & Live load shear in interval 2 & $183.39 \times 10^{3}$ & 0.243 \\
$P_{S 3}(\mathrm{~N})$ & Live load shear in interval 3 & $228.51 \times 10^{3}$ & 0.243 \\
$W\left(\mathrm{~N} / \mathrm{m}^{3}\right)$ & Self weight of concrete & $22.74 \times 10^{3}$ & 0.10 \\
\hline
\end{tabular}

The optimization problem is formulated as a CRBDO problem with four reliability components. The objective of the optimization is to minimize the initial cost of the girder and the initial cost is defined as:

$$
c_{0}(\mathbf{x})=0.75 C_{s} L_{g} A_{s}+C_{s} n_{s} A_{v}\left(h_{f}+h_{w}-\alpha+0.5 b_{w}\right)+C_{c} L_{g}\left(b h_{f}+b_{w} h_{w}\right)
$$

where $C_{s}=50$ and $C_{c}=1$ are the unit price of steel and concrete; $L_{g}=18.30$ is the total length of the girder with unit of meters; $n_{s}=\left(1 / S_{1}+1 / S_{2}+1 / S_{3}\right) L_{g} / 3$ is the total number of stirrups; and $\alpha=0.1 \mathrm{~m}$ is the distance from the bottom fiber to the centroid of tension reinforcement as shown in Fig. 12. The constraints are per the design specification by AASHTO [34] with some other physical limitations. There are four probabilistic constraints with each of them corresponding to one possible failure mode of the girder. The four failure modes considered in this problem are flexural failure at midspan and shear failure at the intervals 1, 2, and 3 which are marked as I-1, I-2, and I-3 in Fig. 13. The threshold of failure probability are the same for all of the four limit states, which is taken as $P_{f}^{t}=0.001350$ (equivalent to $\beta^{t}=3.0$ ). Readers are referred to [33] for the expressions of the limit state functions as well as other 23 deterministic constraints. By definition, the four limit state 


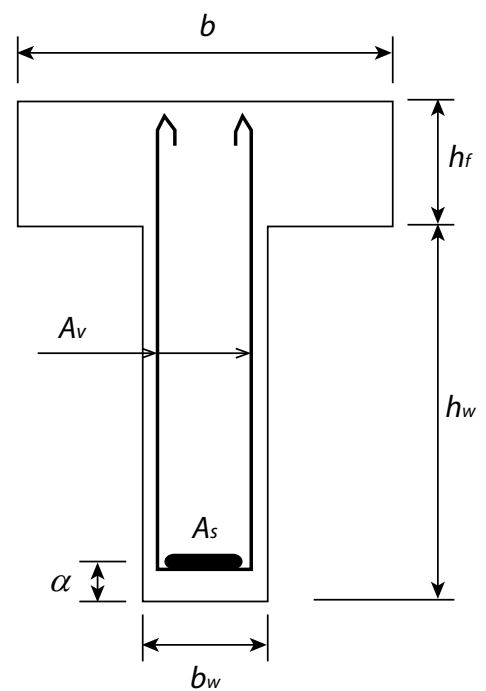

Figure 12: Cross section of reinforced concrete girder.

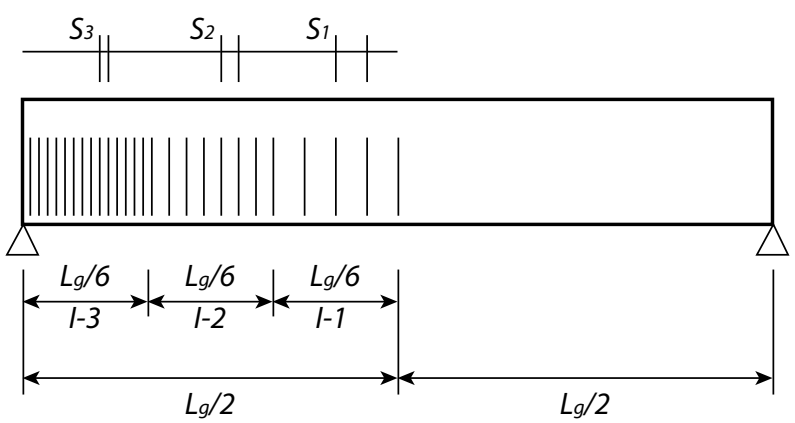

Figure 13: Shear reinforcement arrangement in reinforced concrete girder.

functions are all explicit nonlinear functions of the design variables and random variables. Some of the design formula provided by AASHTO are not continuous functions due to the fact that the plastic neutral axis can be either in the flange or web of the cross section. Following the procedure implemented by Royset and Polak [6], the optimization is performed individually for each case and the one that yields minimal initial cost is taken as the solution.

The problem is solved in [6] using a sample average approximation (SAA) which employs Monte Carlo method to estimate the failure probability and its gradient. The accuracy of the approximation is gradually improved during the optimization process due to the increasing number of sampling. In this paper, we solve the same problem using $P_{f}$ and $\nabla_{\mathbf{x}} P_{f}$ approximated by the proposed SML method with orthogonal fitting scheme. The design point determined by the improved HLRF algorithm [30] is taken as the reference point. The initial 

in MATLAB [35] optimization toolbox using the "active-set" strategy. The optimization converges to an optimal design with 14 iterations in 35.47 CPU seconds as reported by MATLAB profiler on a laptop with a $2.30 \mathrm{GHz} \mathrm{CPU}$. The convergence of objective function is shown in Fig. 14. Table. 4 lists the obtained optimal design. The result is compared with the result computed by SAA method in [6] as shown in Table. 4. We can observe that the two design are quite close to each other with the design by SML method possessing a slightly smaller objective. The main difference is the design of shear reinforcement where the final design by the new method yields a smaller area per stirrup but also smaller spacings between them (i.e. $S_{1}, S_{2}$ and $S_{3}$ ). Furthermore, plain MCS with c.o.v. $=0.05$ is performed to check the failure probability of each failure modes of the optimal design by SML method, and the obtained failure probabilities are 0.001256, 0.001408, 0.001381, and 0.001309 which are all very close to the target probability of failure $P_{f}^{t}$. Thus eventually the two designs yield similar behavior and cost. One possible reason for the small differences could be that in the sample average approximation approach, for more than half of the total iterations, the sensitivity is estimated only based on 40 simulations which could be inaccurate, thus the optimization can be lead to the neighborhood of a local minimum which cannot be further improved by increasing the accuracy of the sensitivity estimation.

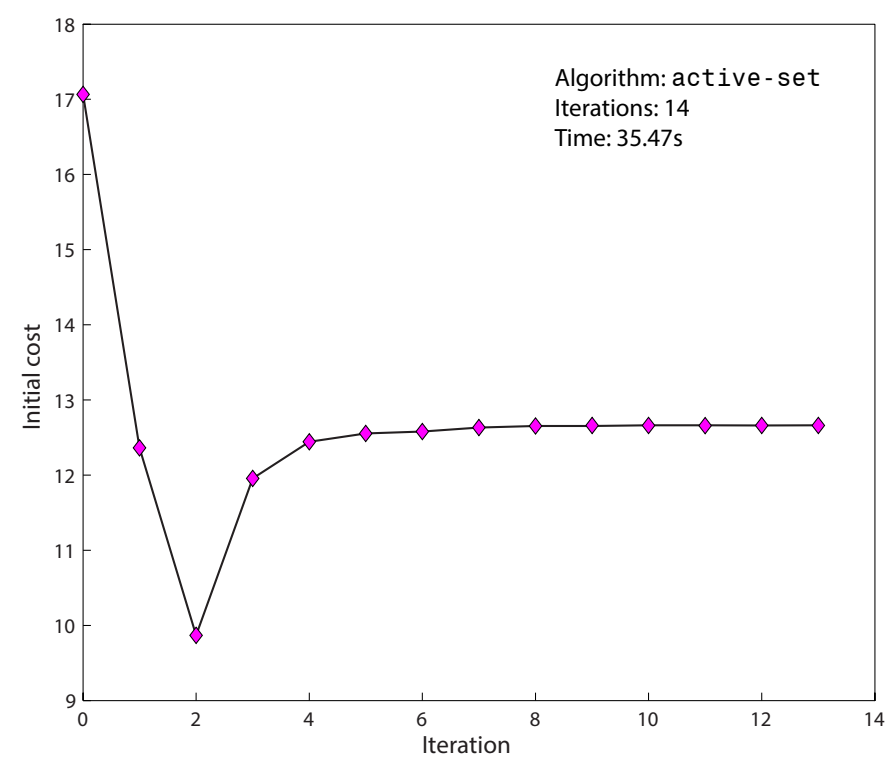

Figure 14: Convergence in objective $c_{0}$. 
Table 4: Optimal Design of Reinforced Concrete Girder

\begin{tabular}{cccc}
\hline Parameter & Design by SAA* & Design by SML & Difference $(\%)$ \\
\hline$A_{s}\left(\mathrm{~m}^{2}\right)$ & 0.008954 & 0.008916 & -0.4412 \\
$b(\mathrm{~m})$ & 0.384 & 0.379 & -1.2895 \\
$h_{f}(\mathrm{~m})$ & 0.411 & 0.415 & 0.9794 \\
$b_{w}(\mathrm{~m})$ & 0.197 & 0.196 & -0.3839 \\
$h_{w}(\mathrm{~m})$ & 0.789 & 0.785 & -0.5102 \\
$A_{v}\left(\mathrm{~m}^{2}\right)$ & 0.0001685 & 0.0001555 & -7.7398 \\
$S_{1}(\mathrm{~m})$ & 0.535 & 0.457 & -14.5762 \\
$S_{2}(\mathrm{~m})$ & 0.230 & 0.204 & -11.2639 \\
$S_{3}(\mathrm{~m})$ & 0.143 & 0.129 & -10.0270 \\
$c_{0}$ & 12.696 & 12.660 & -0.2859 \\
\hline
\end{tabular}

*Note: The data for the design by sample average approximation (SAA) method is collected from [6]

The MATLAB profiler shows that the total number that the SML subroutine being called by the main function is only 144 times (36 for each reliability component), which means happened at most $2 \times 8 \times 144=2304$ times. Comparing to the large number of gradient evaluations required by the sample average approximation method, the proposed method shows a significant advantage in computational cost. It should also be noted that actually the sub-function of improved HLRF algorithm for finding the design point spends up to $79.74 \%$ of the total computation time, and the function of SML method itself only takes a small portion of time.

\subsection{Application to reliability-based topology optimization}

In this example, we are going to apply the proposed method to reliability-based topology optimization (RBTO) problem on ground structure [27, 36, 28]. The ground structure approach is one way to do topology optimization which, is particularly good for optimal designs of truss layouts. The optimal topology is extracted from a very dense set of potential joints 
and bars by sizing the members and allowing them to vanish. In this kind of problems, the evaluation of the gradient of the limit state function with respect to design variables involves finite element analysis which is expensive to compute, thus a MCS-based method is not suitable. On the other hand, the solution of optimal topology is quite sensitive to the gradient information, which requires us to provide approximations with good accuracy. The objective is to minimize the volume of the structure with a probabilistic constraint described in terms of compliance. The problem formulation is given as follows:

$$
\begin{gathered}
\min _{\mathbf{x}} V=\mathbf{L}^{\mathrm{T}} \mathbf{x} \\
\text { s.t. } \quad P\left(C^{\text {max }}-C(\mathbf{x})<0\right)-P_{f}^{t} \leqslant 0 \\
\mathbf{x}_{\min } \leqslant \mathbf{x} \leqslant \mathbf{x}_{\max }
\end{gathered}
$$

where $\mathbf{L}$ is a vector of element length; and the design variables $\mathbf{x}$ are member areas of the ground structure. The limit state function specifies a threshold on the compliance of structure. Compliance is the inner product of the external force vector $\mathbf{F}$ and the nodal displacement vector $\mathbf{d}$, that is $C=\mathbf{F}^{\mathrm{T}} \mathbf{d}$, which is reciprocal to the global stiffness of a structure. The displacement field $\mathbf{d}$ is related to member areas through the equilibrium equation $\mathbf{K}(\mathbf{x}) \mathbf{d}=\mathbf{F}$, where $\mathbf{K}$ is the stiffness matrix. $\mathbf{x}_{\min }$ and $\mathbf{x}_{\max }$ are the lower and upper bounds of member areas. A small value $10^{-4}$ is assigned to $\mathbf{x}_{\min }$ in order to prevent singularity of the stiffness matrix $\mathbf{K}[27$.

We consider a crane arm design (i.e., the design of the top, horizontal portion of a crane) in a domain shown in Fig. 15. The topology optimization is performed on a $9 \times 3$ ground structure with level 6 nodal connectivity as shown in Fig. 16. The material of the structure is linear elastic with Young's Modulus $E=100$. The structure is subject to two independent vertical loads acting on the two tips of the crane. Each of them is assumed to follow the same marginal normal distribution with mean of 7.0 and standard deviation 3.0. The limit on compliance is set to be $C^{\max }=1.2$, thus the limit state function becomes $G(\mathbf{u}, \mathbf{x})=$ $1.2-\mathbf{F}(\mathbf{u})^{\mathrm{T}} \mathbf{d}(\mathbf{x})$ where $\mathbf{u}$ is the vector of transformed random variables.

A FORM-based single loop PMA is first applied to the problem. This approach allows the MPP to be approximated by non-iterative process. The target failure probability is 


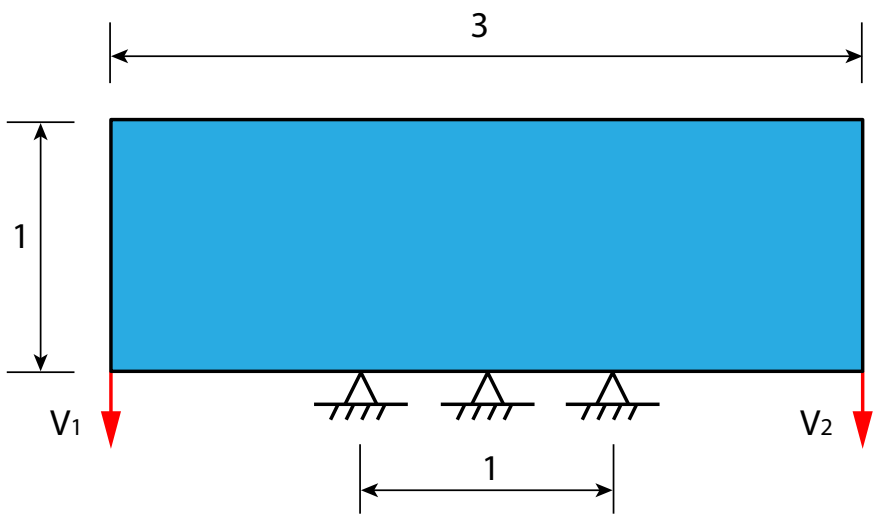

Figure 15: Design domain and boundary conditions of example 2.

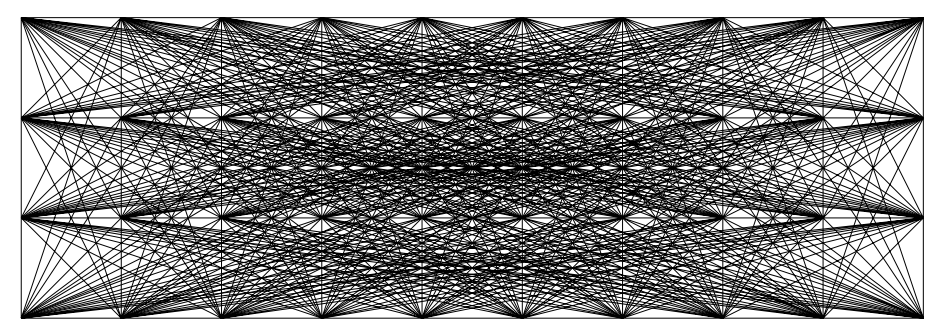

Figure 16: Ground structure used to do RBTO which has 440 non-overlapping members.

$P_{f}^{t}=0.0013$ (i.e. $\beta^{t}=3.0$ ). Fig. 17 shows the obtained optimal design. The result has an optimal volume equals to a dimensionless number 89. The actual failure probability is computed after the optimization by MCS with c.o.v. of 0.05 to be $P_{f, M C S}=0.6554$ which is significantly more than the target value. The design loads and domain are all symmetric thus the optimal topology is expected be symmetric. However, the obtained numerical solution is apparently not symmetric in topology. This is because of the accumulation of error in the sensitivity calculation during the optimization process. For this method, even if we make the

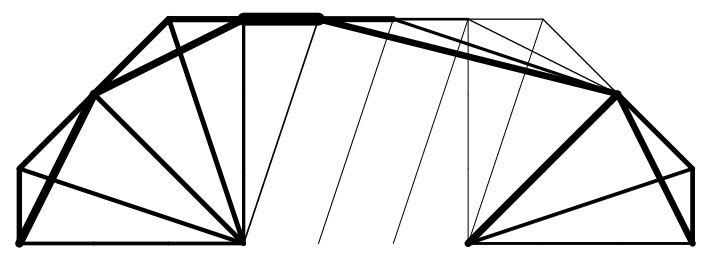

Figure 17: Optimal topology by FORM-based single loop PMA. 
failure probability estimation very accurate using other reliability methods, due to the error in the FORM-based approximation of sensitivity, it is likely impossible to converge to the optimal design. If FORM-based RIA is adopted, the optimization will not converge since the design point obtained by HLRF method will oscillate among iterations of the optimization.

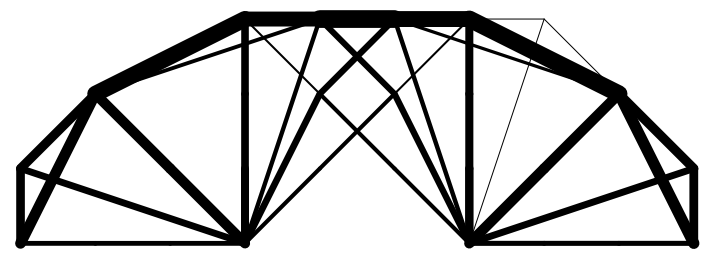

(a)

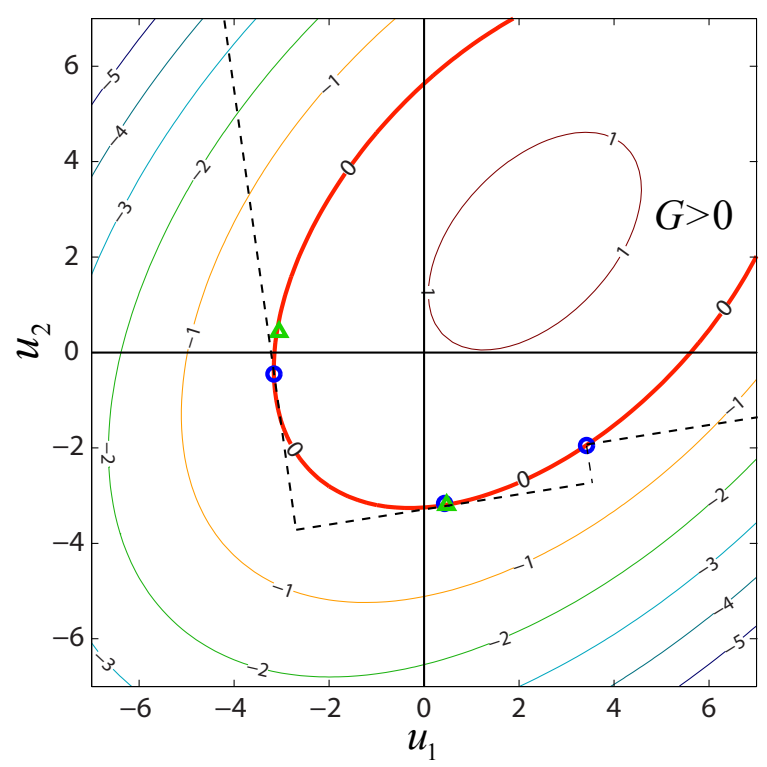

(c)

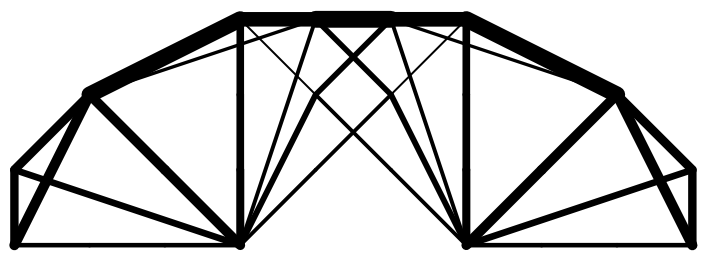

(b)

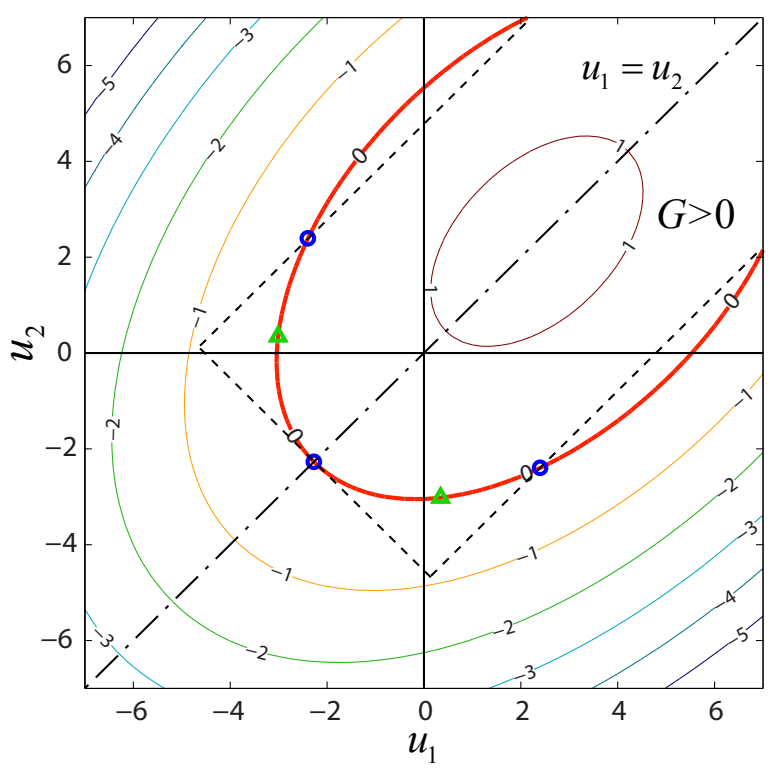

(d)

Figure 18: Optimal topology by SML-based RIA with reference point being (a) the design point and (b) the point on the line $u_{1}=u_{2}$. (c) and (d) shows the contour plot of limit state function for optimal designs shown in (a) and (b) respectively. The blue circles are the fitting points; the green triangles refer to the design points of the final design; the red solid curve is the limit state surface. The black dashed line illustrates the fitting scheme.

Based on the approximation of both $P_{f}$ and $\nabla_{\mathbf{x}} P_{f}$ by the method of SML, RIA is adopted to solve the problem. For this problem since we can expect that the limit state function will have two design points that are symmetric about the line $u_{1}=u_{2}$, we consider two variations of the SML method: (1) orthogonal fitting with design point as the reference point; and (2) 
orthogonal fitting with the reference point to be in the direction of $u_{1}=u_{2}$. The optimal structural layout obtained by variations (1) and (2) are presented in Fig. 18a and 18b. The two results are almost the same in terms of topology. The first one has an optimal volume equals 122 with reliability index of 2.84 computed by post-optimization MCS while the second one has a volume of 114 and reliability index of 2.76. The second design of the SML method converges to a symmetric optimal topology, because by specifying the reference point to be on that potential symmetry axis, the accuracy of the approximation is improved a little comparing to the first one. Fig. $18 \mathrm{c}$ and $18 \mathrm{~d}$ show the contour plots of the limit state functions for the obtained optimal designs. The fitting schemes are shown in dashed lines. The final topologies present very reasonable structural layout, indicating for both variations of the SML, we obtain quite accurate approximations of the failure probability and its sensitivity.

\section{Concluding Remarks and Extension}

This paper proposes the so-called method of segmental multi-point linearization or SML for fast and accurate numerical approximation of the sensitivity of failure probability with respect to design parameters. An approximation of the sensitivity based on the FORM is typically used. However, although the approximation requires very low computational cost, it is often not sufficiently accurate. Approximations of the sensitivity based on MCS can be more accurate but this approach is limited by the expensive computational cost. This paper fills this gap by providing a method that can provide relatively accurate approximations of the parameter sensitivity of the failure probability without requiring high computational cost (see Fig. 1).

The method takes a segmental linear fitting of the limit state function, and constructs the approximation based on the analytical integral expression of the gradient of the failure probability. The method represents a general framework and, as such, could have many variations that provide approximations with different accuracy depending on how the limit state surface is fitted. The paper presents a few possible fitting schemes including tangent fitting, step fitting, and orthogonal fitting. Among these fitting schemes, the orthogonal fitting SML method is recommended for RBDO applications due to its generality, simplicity and accuracy. 
The other two fitting schemes, however, have the property of scalability, meaning that the accuracy of the approximation can be adjusted by adding or removing fitting points. Numerical examples are given to compare the accuracy of the approximations by different methods and different fitting schemes of the proposed method. All the three variations of the SML method present large improvements in terms of accuracy when compared to FORM-based approximations.

The proposed SML method has potential to be further improved in the future by developing new fitting schemes. Within the general proposed SML method, other fitting schemes may be developed to provide more accurate approximations than the currently proposed fitting schemes. In particular, the current fitting schemes of our approach assumes that the limit state function is smooth and continuous with repect to both random and design variables. To apply the piece-wise fitting idea to address system reliability problems, difficulties arise because the intersection of the limit state surfaces might be no longer smooth and continous about the random variables and design variables, which has to be taken into account when we generate the fitting hyperplanes. This is a topic for future work.

In conclusion, we expect the SML method to be useful in the RBDO field, especially for structural optimization, and whenever the gradient of the failure probability needs to be computed with good accuracy given limited computational resource (see Fig. 1).

\section{Acknowledgement}

We acknowledge support from the US NSF (National Science Foundation) through Grants 1321661 and 1437535. In addition, Ke Liu acknowledges support of the China Scholarship Council (CSC), and Glaucio H. Paulino acknowledges support of the Raymond Allen Jones Chair at the Georgia Institute of Technology. The authors would like to extend their appreciation to Prof. Krister Svanberg for providing a copy of his MMA (Method of Moving Asymptotes) code which was used to solve the optimization problem in the last example of this paper. 


\title{
Appendix A. Nomenclature
}

\author{
Abbreviations \\ c.o.v. Coefficient of variation \\ ${ }_{560}$ CDF Cumulative Distribution Function \\ CRBTO Component Reliability-Based Topology Optimization \\ FORM First Order Reliability Method \\ HLRF Hassofer-Lind-Rackwitz-Fiessler (Algorithm) \\ KKT Karush-Kuhn-Tucker (Optimality Conditions) \\ MCS Monte Carlo Simulation \\ MPP Most Probable Point \\ OF Orthogonal Fitting (Scheme) \\ PDF Probability Density Function \\ PMA Performance Measure Approach \\ 570 RBDO Reliability-Based Design Optimization \\ RBTO Reliability-Based Topology Optimization \\ RIA Reliability Index Approach \\ SAA Sample Average Approximation \\ SF $\quad$ Step Fitting (Scheme) \\ SML Segmental Multi-point Linearization \\ SORM Second Order Reliability Method \\ SRBTO System Reliability-Based Topology Optimization
}


TF Tangent Fitting (Scheme)

\section{Symbols}

${ }_{580} \quad \bar{G}_{i} \quad$ The affine function describing hyperplane segment $i$

$\bar{S} \quad$ Piecewise linearized limit state surface

$\bar{S}_{i} \quad$ Hyperplane segments of a piecewise linearized limit state surface

$\beta \quad$ Reliability index

$\beta^{t} \quad$ Target reliability index

$\beta_{1} \quad$ FORM approximation of reliability index

$\Delta D \quad$ Change of failure domain

$\lambda, \gamma_{i} \quad$ Lagrange multipliers

u $\quad$ Reduced random variables

$\mathbf{e}_{i} \quad$ Orthonormal basis of space

${ }_{590} \mathbf{n}_{i} \quad$ Normal direction of hyperplane segment $i$

Q Rotational Operator

$\mathbf{u}^{*} \quad$ Most likely failure point (design point)

$\mathbf{u}^{t} \quad$ MPP

$\mathbf{v}, \mathbf{u} \quad$ Random variables and the transformed random variables

x Design variables

$\Phi, \varphi \quad$ CDF and PDF of standard normal distribution

$b_{i} \quad$ The distance from the origin to the hyperplane that contains segment $i$

$f_{\mathbf{v}}(\mathbf{v}) \quad$ Joint PDF of random variables 
$g, G \quad$ Limit state function in original random space and transformed random space

${ }_{600} h_{i} \quad$ Deterministic constraints

$k_{1}, k_{2}, \eta \quad$ User defined parameters of SML-OF

$n \quad$ Number of random variables

$P_{f} \quad$ Failure probability

$P_{f}^{t} \quad$ Target failure probability

$S \quad$ Limit state surface

T Probability preserving transformation

$W_{i} \quad$ Weight for contribution of segment $i$

\section{Appendix B. A brief derivation of the parameter sensitivity of the failure prob- ability}

In this section, we will derive the analytical expression for the sensitivity of failure probability with respect to design parameters. Further derivations can be found in reference [3] and [4]. Assume that the limit state function is continuous and differentiable to first order. If we take a perturbation in $x_{i}$ (denoted as $\delta x_{i}$ ) on the design variables, the limit state surface would evolve to a new shape in the random variable space. If the movement of each point on the limit state surface is denoted as $\delta \mathbf{u}$, the following equation describes the motion:

$$
\delta G(\mathbf{u}, \mathbf{x})=\frac{\partial G(\mathbf{u}, \mathbf{x})}{\partial x_{i}} \delta x_{i}+\nabla_{\mathbf{u}}^{\mathrm{T}} G(\mathbf{u}, \mathbf{x}) \delta \mathbf{u}=0
$$

The limit state surface in the $\mathbf{u}$-space can be regarded as a level set of the surface $G(\mathbf{u}, \mathbf{x})=$ 0 in the hybrid space of random variables $\mathbf{u}$ and design variables $\mathbf{x}$ at a certain level of $x_{i}$. Each coordinate of $\mathbf{x}$ is orthogonal to $\mathbf{u}$ in the hybrid space since we assume that the random variables are independent from the design variables. Hence, only the normal component of $\delta \mathbf{u}$, which is due to the perturbation $\delta x_{i}$, would contribute to the propagation of limit state surface. The normal component of $\delta \mathbf{u}$ is along the same direction of $\nabla_{\mathbf{u}} G(\mathbf{u}, \mathbf{x})$. We rotate 


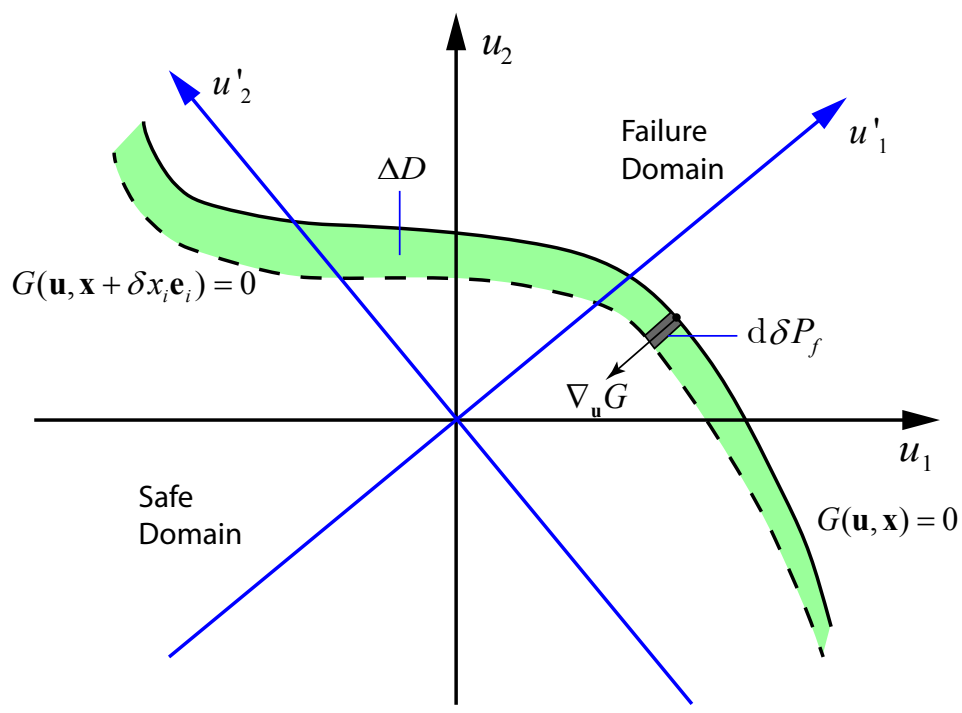

Figure B.19: Geometric representation of a general continuous limit state surface.

the coordinate system of the $\mathbf{u}$-space such that the axis $\mathbf{e}_{1}^{\prime}$ is in the opposite direction of $\nabla_{\mathbf{u}} G(\mathbf{u}, \mathbf{x})$. Let's denote the new coordinates as $\mathbf{u}^{\prime}=\mathbf{Q u}=\left[u_{1}^{\prime}, \hat{\mathbf{u}}^{\prime}\right]^{\mathrm{T}}$, where the rotational operator $\mathbf{Q}$ should be a function of $\mathbf{u}$ and $\mathbf{x}$. Notice that $\mathbf{Q}$ is not the same for different points on limit state surface. Furthermore, we can always properly choose the rotational operator such that it is in the special orthogonal group, which contains the orthogonal matrices of determinant 1. Then Eq. (B.1) can be rewritten as:

$$
\delta G(\mathbf{u}, \mathbf{x})=\frac{\partial G(\mathbf{u}, \mathbf{x})}{\partial x_{i}} \delta x_{i}+\left\|\nabla_{\mathbf{u}} G(\mathbf{u}, \mathbf{x})\right\| \delta u_{1}^{\prime}=0
$$

The change of failure probability due to a perturbation of $\mathbf{x}$ is the integration of the probability density function (PDF) over the change of the failure domain which is denoted as $\Delta D$, as shown in the shaded area in Fig. B.19. Therefore:

$$
\delta P_{f}=\int_{G\left(\mathbf{u}, \mathbf{x}+\delta x_{i}\right) \leqslant 0} \varphi_{n}(\mathbf{u}) \mathrm{d} \mathbf{u}-\int_{G(\mathbf{u}, \mathbf{x}) \leqslant 0} \varphi_{n}(\mathbf{u}) \mathrm{d} \mathbf{u}=\int_{\Delta D} \varphi_{n}(\mathbf{u}) \mathrm{d} \mathbf{u}
$$

Taking a small piece of the $\Delta D$ and calculating the volume under the probability density function, we obtain:

$$
\mathrm{d} \delta P_{f}=\varphi_{n}\left(\mathbf{u}^{\prime}\right) \delta u_{1}^{\prime} \delta \hat{\mathbf{u}}^{\prime}
$$


where $\delta \hat{\mathbf{u}}^{\prime}=\delta u_{2}^{\prime} \delta u_{3}^{\prime} \ldots \delta u_{n}^{\prime}$ and $n$ is the number of random variables. Substituting (B.2) into (B.4), we establish that:

$$
\mathrm{d} \delta P_{f}=-\varphi_{n}\left(\mathbf{u}^{\prime}\right) \frac{\partial G}{\partial x_{i}} \frac{\delta x_{i}}{\left\|\nabla_{\mathbf{u}} G\right\|} \delta \hat{\mathbf{u}}^{\prime}
$$

Taking the limit $\delta \mathbf{u} \rightarrow 0$ and integrating $\mathrm{d} \delta P_{f}$ over the limit state surface, an integral expression for the change of failure probability is obtained:

$$
\delta P_{f}=\int_{S^{\prime}} \mathrm{d} \delta P_{f}=\int_{S^{\prime}}-\varphi_{n}\left(\mathbf{u}^{\prime}\right) \frac{\partial G}{\partial x_{i}} \frac{\delta x_{i}}{\left\|\nabla_{\mathbf{u}} G\right\|} \mathrm{d} \hat{\mathbf{u}}^{\prime}
$$

where $S$ and $S^{\prime}$ are the limit state surface in original coordinates and rotated coordinates. Rearranging the terms, we get the sensitivity of the failure probability with respect to design variables as:

$$
\frac{\partial P_{f}}{\partial x_{i}}=\frac{\delta P_{f}}{\delta x_{i}}=\int_{S^{\prime}}-\varphi_{n}\left(\mathbf{u}^{\prime}\right) \frac{\partial G}{\partial x_{i}} \frac{1}{\left\|\nabla_{\mathbf{u}} G\right\|} \mathrm{d} \hat{\mathbf{u}}^{\prime}=\int_{S^{\prime}}-\frac{\varphi_{n}\left(\mathbf{u}^{\prime}\right)}{\left\|\nabla_{\mathbf{u}} G\right\|} \frac{\partial G}{\partial x_{i}} \mathrm{~d} S^{\prime}
$$

${ }_{640}$ A transformation of the coordinates should satisfies $\mathrm{d} S^{\prime}=(\operatorname{det} \mathbf{Q})\left\|\mathbf{Q}^{-T} \mathbf{n}\right\| \mathrm{d} S$, where $\mathbf{n}$ is the normal direction of the infinitesimal piece of surface. Although $\mathbf{Q}$ is not a constant operator, its determinant always equals 1 , and $\left\|\mathbf{Q}^{-T} \mathbf{n}\right\|$ also remains 1 . Furthermore, due to the rotational symmetry of the standard normal space, $\varphi_{n}\left(\mathbf{u}^{\prime}\right)=\varphi_{n}(\mathbf{u})$. Thus Eq. (B.7) can be rewritten in the original coordinate as

$$
\frac{\partial P_{f}}{\partial x_{i}}=-\int_{S} \frac{\varphi_{n}(\mathbf{u})}{\left\|\nabla_{\mathbf{u}} G\right\|} \frac{\partial G}{\partial x_{i}} \mathrm{~d} S
$$

Applying Eq. (B.8) to each component of $\mathbf{x}$, we can assembly the gradient vector as:

$$
\nabla_{\mathbf{x}} P_{f}=-\int_{S} \frac{\varphi_{n}(\mathbf{u})}{\left\|\nabla_{\mathbf{u}} G\right\|} \nabla_{\mathbf{x}} G \mathrm{~d} S
$$

Eq. (B.9) can also be found in reference [3] and [6]. A complete mathematical derivation for the sensitivity of probability integral with respect to all kinds of parameters can be found in the paper by Uryasev [4] published in 1994. 


\section{Appendix C. Pseudo code for orthogonal fitting SML}

650 function $\mathrm{SMLOF}$ (reference point $\mathbf{u}_{1}$, limit state function $G(\mathbf{u}, \mathbf{x})$, current design $\mathbf{x}$ )

//Initialize parameters

$b_{1}=\left\|\mathbf{u}_{1}\right\|$

$k_{1}=\sqrt{-2 \ln (0.1)+b_{1}^{2}} / b_{1}, \eta=0.7, k_{2}=\min \left(1,3 / b_{1}\right)$

$\mathbf{e}_{1}^{\prime}=\mathbf{u}_{1} /\left\|\mathbf{u}_{1}\right\|$

Find new orthogonal basis $\mathbf{e}_{1}^{\prime}, \mathbf{e}_{2}^{\prime}, \ldots, \mathbf{e}_{n}^{\prime}$.

$/ /$ Assume $G(\mathbf{0}, \mathbf{x})>0$

//Search the positive directions

for $i=2,3, \ldots, n$ do

if $G\left(k_{1} b_{1} \mathbf{e}_{i}^{\prime}, \mathbf{x}\right)<0$ then

660

find $b_{i}>0$ that solves $G\left(b_{i} \mathbf{e}_{i}^{\prime}, \mathbf{x}\right)=0$

$$
\begin{aligned}
\mathbf{u}_{i} & =b_{i} \mathbf{e}_{i}^{\prime} \\
c_{i} & =\Phi\left(-b_{i}\right), p_{i}=0
\end{aligned}
$$

else

find $b_{i}>0$ that solves $G\left(k_{2} b_{1} \mathbf{e}_{i}^{\prime}+b_{i} \mathbf{e}_{1}^{\prime}, \mathbf{x}\right)=0$

$$
\begin{aligned}
& \mathbf{u}_{i}=k_{2} b_{1} \mathbf{e}_{i}^{\prime}+b_{i} \mathbf{e}_{1}^{\prime} \\
& c_{i}=0, p_{i}=\Phi\left(-\eta k_{2} b_{1}\right) \quad / / \text { Partition }
\end{aligned}
$$

end if

end for

//Search the negative directions

for $i=1,2, \ldots, n$ do

$$
\text { if } G\left(-k_{1} b_{1} \mathbf{e}_{i}^{\prime}, \mathbf{x}\right)<0 \text { then }
$$

find $b_{i+n}>0$ that solves $G\left(-b_{i+n} \mathbf{e}_{i}^{\prime}, \mathbf{x}\right)=0$

$$
\begin{aligned}
& \mathbf{u}_{i+n}=-b_{i+n} \mathbf{e}_{i}^{\prime} \\
& c_{i+n}=\Phi\left(-b_{i+n}\right), p_{i+n}=0
\end{aligned}
$$

else if $i=1$ then

$$
\begin{aligned}
& \mathbf{u}_{i+n}=-\mathbf{e}_{i}^{\prime} \\
& c_{i+n}=0, p_{i+n}=0
\end{aligned}
$$

//This is not an active fitting point, the corresponding weight will be 0 
else

find $b_{i+n}>0$ that solves $G\left(-k_{2} b_{1} \mathbf{e}_{i}^{\prime}+b_{i+n} \mathbf{e}_{1}^{\prime}, \mathbf{x}\right)=0$

$$
\begin{aligned}
& \mathbf{u}_{i+n}=-k_{2} b_{1} \mathbf{e}_{i}^{\prime}+b_{i+n} \mathbf{e}_{1}^{\prime} \\
& c_{i+n}=0, p_{i+n}=\Phi\left(-\eta k_{2} b_{1}\right)
\end{aligned}
$$

end if

end for

$/ /$ First approximation of $P_{f}$

$P_{f}=1-\prod_{k=1}^{n}\left(1-c_{k}-c_{k+n}\right)$

//Compute weights for plane segments determined by intersection fitting points

for $j=1,2, \ldots, 2 n$ do

$$
i=(j-1) \% n+1
$$

$$
\text { if } c_{j} \neq 0 \text { then }
$$

$$
W_{j}=-\varphi\left(b_{j}\right) \prod_{k=1, k \neq i}^{n}\left(1-c_{k}-c_{k+n}\right) /\left\|\nabla_{\mathbf{u}} G\left(\mathbf{u}_{j}, \mathbf{x}\right)^{\mathrm{T}} \mathbf{e}_{i}^{\prime}\right\|
$$

\section{end if}

\section{end for}

//Compute weights for plane segments determined by off-axis fitting points

for $j=2,3, \ldots, 2 n$ do

$$
i=(j-1) \% n+1
$$

$$
\text { if } c_{j}=0 \text { then }
$$

$$
\begin{aligned}
& W_{j}=-\varphi\left(b_{j}\right) p_{j} \prod_{k=2, k \neq i}^{n}\left(1-c_{k}-c_{k+n}\right) /\left\|\nabla_{\mathbf{u}} G\left(\mathbf{u}_{j}, \mathbf{x}\right)^{\mathrm{T}} \mathbf{e}_{1}^{\prime}\right\| \\
& P_{f}=P_{f}+\left(\Phi\left(-b_{j}\right)-c_{1}\right) \prod_{k=2, k \neq i}^{n}\left(1-c_{i}-c_{i+n}\right) \quad / / \text { Update approximation of } P_{f}
\end{aligned}
$$

$$
c_{j}=p_{j} \quad / / \text { Avoid overlapping }
$$

end if

end for

$\nabla_{\mathbf{x}} P_{f}=\sum_{k=1}^{2 n} W_{k} \nabla_{\mathbf{x}} G\left(\mathbf{u}_{k}, \mathbf{x}\right)$

return $\nabla_{\mathbf{x}} P_{f}$ and $P_{f}$

end function 


\section{References}

[1] O. Ditlevsen, H. O. Madsen, Structural Reliability Methods, John Wiley \& Sons Inc, 1996.

[2] M. Hohenbichler, R. Rackwitz, Sensitivity and importance measures in structural reliability, Civil Engineering Systems 3 (4) (1986) 203-209.

[3] K. Breitung, Parameter sensitivity of failure probabilities, in: A. Der Kiureghian, P. Thoft-Christensen (Eds.), Reliability and Optimization of Structural Systems 90, Vol. 61 of Lecture Notes in Engineering, Springer Berlin Heidelberg, 1991, pp. 43-51.

[4] S. Uryasev, Derivatives of probability functions and integrals over sets given by inequalities, Journal of Computational and Applied Mathematics 56 (12) (1994) 197 - 223.

[5] A. Karamchandani, C. Cornell, Sensitivity estimation within first and second order reliability methods, Structural Safety 11 (2) (1992) 95 - 107.

[6] J. Royset, E. Polak, Reliability-based optimal design using sample average approximations, Probabilistic Engineering Mechanics 19 (4) (2004) 331 - 343.

[7] M. Fu, Stochastic gradient estimation, in: S. Henderson, B. Nelson (Eds.), Handbook on Operations Research and Management Science: Simulation, Elsevier, 2006.

[8] J. Royset, E. Polak, Extensions of stochastic optimization results from problems with simple to problems with complex failure probability functions, Journal of Optimization Theory and Applications 133 (1) (2007) 1-18.

[9] J. Royset, E. Polak, Sample average approximations in reliability-based structural optimization: Theory and applications, in: Y. Tsompanakis, N. Lagaros (Eds.), Structural Design Optimization Considering Uncertainties, Springer, New York, 2008.

[10] W. van Ackooij, R. Henrion, Gradient formulae for nonlinear probabilistic constraints with gaussian and gaussian-like distributions, SIAM Journal on Optimization 24 (4) $730 \quad$ (2014) 1864-1889. 
[11] R. Rackwitz, Risk control and optimization for structural facilities, in: E. Sachs, R. Tichatschke (Eds.), System Modeling and Optimization XX, Vol. 130 of IFIP The International Federation for Information Processing, Springer US, 2003, pp. 143-167.

[12] J. Tu, K. K. Choi, Y. H. Park, Design Potential Method for Robust System Parameter Design, AIAA Journal 39 (2001) 667-677.

[13] G. Cheng, L. Xu, L. Jiang, A sequential approximate programming strategy for reliability-based structural optimization, Computers and Structures 84 (21) (2006) 1353 $-1367$.

[14] J. Liang, Z. P. Mourelatos, E. Nikolaidis, A single-loop approach for system reliabilitybased design optimization, Impact of Computing in Science and Engineering 129 (12) (2007) $1215-1224$.

[15] M. McDonald, S. Mahadevan, Design optimization with system-level reliability constraints, Journal of Mechanical Design 130 (2) (2008) 021403-1 - 021403-10.

[16] T. H. Nguyen, J. Song, G. H. Paulino, Single-loop system reliability-based design optimization using matrix-based system reliability method: Theory and applications, Journal of Mechanical Design 132 (1) (2010) 011005-1 - 011005-11.

[17] J. Royset, A. Kiureghian, E. Polak, Reliability-based optimal design of series structural systems, Journal of Engineering Mechanics 127 (6) (2001) 607-614.

[18] J. Royset, A. Kiureghian, E. Polak, Reliability-based optimal structural design by the decoupling approach, Reliability Engineering \& System Safety 73 (3) (2001) 213 - 221.

[19] J. Royset, A. Der Kiureghian, E. Polak, Optimal design with probabilistic objective and constraints, Journal of Engineering Mechanics 132 (1) (2006) 107-118.

[20] J. L. Beck, E. Chan, A. Irfanoglu, C. Papadimitriou, Multi-criteria optimal structural design under uncertainty, Earthquake Engineering \& Structural Dynamics 28 (7) (1999) 741-761. 
[21] H. Nakamura, A. Miyamoto, K. Kwamura, Optimization of bridge maintenance strategies using GA and IA techniques, in: Proceedings of the ninth IFIP WG7. 5 Working Conference on Reliability and Optimization of Structural Systems, Ann Arbor, Michigan, 2000.

[22] S. Mathakari, P. Gardoni, P. Agarwal, A. Raich, T. Haukaas, Reliability-based optimal design of electrical transmission towers using multi-objective genetic algorithms, Computer-Aided Civil and Infrastructure Engineering 22 (4) (2007) 282-292.

[23] T. H. Nguyen, J. Song, G. H. Paulino, Single-loop system reliability-based topology optimization considering statistical dependence between limit-states, Structural and Multidisciplinary Optimization 44 (5) (2011) 593-611.

[24] A. Der Kiureghian, H. Lin, S. Hwang, Second order reliability approximations, Journal of Engineering Mechanics 113 (8) (1987) 1208-1225.

[25] M. Heath, Scientific Computing: An Introductory Survey, Second Edition, McGraw-Hill, 1997.

[26] K. Liu, Segmental multi-point linearization for topology optimization and reliability analysis, Master's thesis, University of Illinois, Urbana, USA (2014).

[27] P. Christensen, A. Klarbring, An Introduction to Structural Optimization, Solid Mechanics and its Applications, Springer, 2008.

[28] T. Zegard, G. Paulino, GRAND - ground structure based topology optimization for arbitrary 2D domains using MATLAB, Structural and Multidisciplinary Optimization (2014) 1-22.

[29] A. D. Kiureghian, T. Dakessian, Multiple design points in first and second-order reliability, Structural Safety 20 (1) (1998) $37-49$.

[30] Y. Zhang, A. D. Kiureghian, Two improved algorithms for reliability analysis, reliability and optimization of structural systems, in: Proceedings of the Sixth IFIP WG7. 5 Working Conference on Reliability and Optimization of Structural Systems, Assisi, Italy, 1995, pp. 297-304. 
[31] A. Hahnel, P. Franchin, B. Gencturk, J. Song, S. Pakzad, B. Sudret, J. Bourinet, T. Haukaas, Finite element reliability using MATLAB (FERUM) Version 3, University of California, Berkeley, California, 2000.

[32] R. Rackwitz, Reliability analysisa review and some perspectives, Structural Safety 23 (4) (2001) $365-395$.

[33] J. Royset, A. Der Kiureghian, E. Polak, Reliability-based design optimization of series structural systems, Tech. Rep. UCB/SEMM-2002/15, Department of Civil Engineering and Environmental Engineering, University of California, Berkeley, California (2002).

[34] American Association of State Highway and Transportation Officials (AASHTO) 15th Ed., Washington D.C., Standard specifications for highway bridges (1992).

[35] MATLAB version 8.1.0.604 (R2013a), The MathWorks Inc., Natick, Massachusetts, 2013.

[36] K. Mogami, S. Nishiwaki, K. Izui, M. Yoshimura, N. Kogiso, Reliability-based structural optimization of frame structures for multiple failure criteria using topology optimization techniques, Structural and Multidisciplinary Optimization 32 (4) (2006) 299-311. 